$\begin{array}{ll}\text { Italique } & \text { Italique } \\ \text { Poésie italienne de la Renaissance }\end{array}$

XVI | 2013

Varia

\title{
Mostri, menzogne e meraviglie nel Bosadrello di Baldassarre da Fossombrone
}

\section{Giuseppe Crimi}

\section{(2) OpenEdition}

\section{Journals}

\section{Edizione digitale}

URL: http://journals.openedition.org/italique/374

DOI: 10.4000/italique.374

ISSN: 1663-4438

\section{Editore}

Librairie Droz

\section{Edizione cartacea}

Data di pubblicazione: 1 dicembre 2013

Paginazione: 155-191

ISBN: 978-2-600-01779-4

ISSN: 1423-3983

\section{Notizia bibliografica digitale}

Giuseppe Crimi, « Mostri, menzogne e meraviglie nel Bosadrello di Baldassarre da Fossombrone », Italique [Online], XVI | 2013, online dal 01 décembre 2016, consultato il 20 avril 2019. URL : http:// journals.openedition.org/italique/374 ; DOI : 10.4000/italique.374 


\section{G I U S EP PE C R I M I}

MO S T R I, M E N Z O N E E MER A V I G L I E NEL BOSADRELLO

D I B A L A S A R R D A FOS SOMB R O N E 



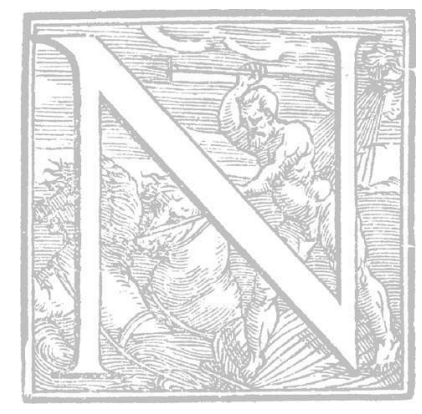

el i888 Vittorio Rossi, in una delle cospicue appendici contenute nell'edizione delle Lettere di Andrea Calmo, segnalò un opuscolo anonimo intitolato Bugiardello, stampato a Venezia da Mattio Pagan negli anni Cinquanta del Cinquecento: il contenuto dell'operetta consisteva in cinquantacinque sonetti caudati di stampo comico, seguiti da vari distici con andamento proverbiale, dei quali Rossi provvide a indicare anche un testi-

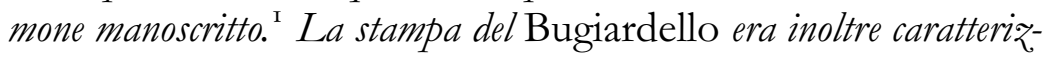
zata da una serie di combinazioni di tre dadi impresse in calce a ciascun sonetto. Si trattava, quindi, di un libretto utilizato come passatempo, per il quale occorre ipotizzare un uso collettivo e in cui il motore era il lancio contemporaneo di tre dadi: per tale ragione l'opuscolo, sbrigativamente etichettato "libro di sorte", fu considerato, anche dagli studiosi successivi, come un testo popolare. ${ }^{2}$ Rossi, giunto alla scoperta grazie al passo di una lettera calmiana, aveva dimostrato che un altro testo chiamato "Bugiardello" da Scipione Casali era in realtà un esemplare dell'edizione del I557 del Libro de la ventura di Lorenzo Spirito (Venezia, Mattio Pagan), mutilo di alcune carte iniziali, compreso il frontespizio: al termine dell'opera, della quale, pertanto, non potevano essere facilmente riconosciuti autore e titolo, era stampata una lettera di tale Luigi Brocardi, che in un passo definiva l'opera stessa "Bugiardello", ${ }_{4}$ da cui l'equivoco del Casali: «Ben vi ricordo sopra ogni altra cosa, ch'è intitolato il Bugiardello, né cosa che lui dica havete a pigliare a petto, come se parlasse sensatamente, perché porta secco il suo titolo» (c. A34v). ${ }^{5}$ Una denominazione non proprio casuale visto che dai torchi del Pagan uscivano nello stesso periodo $i$ sonetti del Bugiardello; in più, la definizione di "Bugiardello" per l'opera dello Spirito rispecchiava la comune opinione intorno ai libri di ventura, ritenuti, appunto, mendaci. ${ }^{6}$ Si pensi che con il medesimo titolo Bugiardello circolò nell'Ottocento una sorta di oracolo galante questo si popolare - nel quale erano contenute domande sull'amore e sulla fortuna, in modo simile alle Sorti di Marcolini. ${ }^{7}$

Nel 1943 Giovanni Crocioni, agevolato dalle esplorazioni di Augusto Vernarecci, presentò al mondo degli studiosi un recupero davvero notevole: ${ }^{8}$ l'anonimo Bugiardello altro non era che un'edizione cinquecen- 
tesca del Menzoniero overamente Bosadrello di Baldassarre da Fossombrone, stampato a Ferrara da Severino da Ferrara nel I475 (e, come scoperto di recente, con la complicità di Felice Feliciano). ${ }^{9}$ Cancelliere e segretario dei Gonzaga, secondo la rubrica dell'incunabolo, Baldassarre visse a Mantova al servizio di Barbara di Brandeburgo e Ludovico II Gonzaga: proprio su richiesta di Barbara erano stati scritti $i$ sonetti (almeno intorno al I460, stando alla testimonianza di una lettera autografa del cancelliere), incentrati in prevalen za sul tema della menzogna, forse una trasposizione della Lügendichtung, nota nella terre germaniche e con attestazioni anche in altre letterature europee, ${ }^{\mathrm{I}}$ in particolare in Francia, dove è documentata dal tardo Duecento la presenza delle fatrasies, dalle quali si sviluppano le chansons de menteries, testi dalle profonde affinità con $i$ sonetti del Nostro. ${ }^{\text {I }}$ Nella cultura peninsulare, invece, abbiamo testimonianze, in gran parte nell'Ottocento e nel Novecento, delle "canzoni alla rovescia", studiate quasi un secolo fa da Giovanni Giannini. ${ }^{\text {I2 }}$

A ben vedere, l'incunabolo che Crocioni collegò al Bugiardello si differenziava da quest'ultimo non solo per la patina linguistica (oltre che per alcune varianti), ma anche per la presenza di altri due sonetti e per l'assenza dei suddetti proverbi in distici, che il folk.lorista marchigiano pubblicò ignorando che un'edizione era stata già offerta da Francesco Novati. ${ }^{13} \mathrm{Ma}$ la differenza sostanziale sembrava nell'impiego del testo, che nella princeps non presentava le combinazioni dei dadi: ${ }^{{ }^{4}} \mathrm{va}$ aggiunto, tuttavia, che ad agevolare l'uso "oracolare" del testo avranno contribuito un paio allusioni tra i versi: VII I2-I4: "Getâr le sorte: a un tocò $i$ meloni, / tartuffe a l'altro e 'l terzo li soletto / in mare tempestoso si adormia» e XLIII I2-I4, dove si racconta di uno specchio magico in grado di rivelare «quel che $i$ cieli dispongon de la terra / $e$ quando serà pace et quando guerra» (I6-I7). Pratiche divinatorie peraltro attestate nella letterature coeva, come in un paio di ottave della Spagna ferrarese. ${ }^{\text {IS }}$

Chi scrive ha procurato il testo critico del Bosadrello fondato sull'appena citato incunabolo stampato da Severino da Ferrara. Nel corso della recensio è stato possibile individuare altri testimoni oltre ai due noti fino al 1943: un'edizione stampata da Giovanni Battista Sessa e priva di data, ma da collocare tra il Is0I e il Isos; un'edizione veneziana di Guglielmo da Fontaneto del I553; e un'edizione impressa da Mattio Pagan, priva di data e differente da quella già nota. A queste 
stampe cinquecentesche ne va aggiunta una, bresciana, del I6IO (Bartolomeo Fontana), in cui i sonetti, insieme con altri cinquantacinque, furono riproposti da Francesco Moise Chersino, il quale si attribui la paternità di tutti i componimenti. ${ }^{16}$

Sarà utile in questa sede tornare, fugacemente, su alcuni sonetti del Bosadrello che meglio rappresentano le peculiarità della raccolta, integrando le note di commento, per dimostrare che - seppur successivamente spacciato come semplice libretto di poesia burlesca - di popolare esso ha ben poco.

I versi di Baldassarre sono incentrati su alcuni dei mirabilia medievali, recuperati in gran parte attraverso $i$ testi che raccontano di un Oriente prodigioso, come il Romanzo d'Alessandro (il condottiero viene citato nel sonetto $\mathrm{IV})^{17}$ e la Lettera del Prete Gianni (il sourano è menzionato nell'attacco del sonetto $L V)^{\mathrm{I} 8}$ o quelli piu propriamente appartenenti alla letteratura odeporica, ${ }^{19}$ tra $i$ quali spiccano alcuni best-seller, ovvero la Navigatio Sancti Brendani, ${ }^{20}$ Odorico da Pordenone e John Mandeville. ${ }^{2 \mathrm{I}}$ In più, l'opera di Pier Candido Decembrio, il De omnium animantium naturis atque formis (ms. Urb. Lat. 276), presentata a Ludovico Gonzaga nel I460, dimostra come un'attenzione nei confronti dei prodotti bizzarri della natura non fosse estranea al gusto mantovano dell'epoca. ${ }^{22}$

L'Oriente era lo specchio rovesciato dell'Occidente, il luogo, acclarato, dove trovavano spazio gli avvenimenti piu sorprendenti, e proprio di Oriente trattano spesso le visioni del Bosadrello. I sonetti raccontano di circostanze relative al paese di Cuccagna, ${ }^{23}$ di situazioni tipiche del mundus inversus, ${ }^{24}$ di episodi di monstra e prodigia. Il monstrum, soprattutto, sin dal medioevo è legato in modo indissolubile all'idea di mendacium. ${ }^{25}$ Seppur dosata con cautela, l'esperienza burchiellesca all'interno dei versi è riconoscibile senza difficoltà; tuttavia rispetto ai testi di riferimento Baldassarre, che intuisce immediatamente le potenzialità della poesia del barbiere di Calimala, compie una scelta del tutto personale, incentrando i versi sul meraviglioso, sul paradossale e sull'iperbolico. ${ }^{26}$

"Quando voleno dire delle gran bosie fingeno il suggetto, o veramente, narran cose tanto da lontano, che testimonio non si trovi per certezæa»: ${ }^{27}$ il passo è tratto dall'Opera nuova, fatica di Angelo Forte, data alle stampe nel I532, che può sintetizzare in modo efficace anche il significato complessivo dei sonetti inclusi nel Bosadrello, intrisi di 
mirabili visioni e di situazioni stupefacenti e fuori dell'ordinario. E si, perché, in sede di racconto, la materia peregrina, non verificabile immediatamente dall'ascoltatore o dal lettore e priva di altre testimonianze, permetteva all'autore di inventare, senza preoccupazioni, o, se non altro, di trascrivere la materia senza il bisogno di un'aderenza scrupolosa alla verità. Il verbo "vedere", elemento caratterizzante dell'obscuritas, ${ }^{28}$ è una costante dei sonetti del Bosadrello, ed è spesso incastonato nell'incipit, altra peculiarità che rimanda sia alla poesia burchiellesca (e fatrasica) ${ }^{29}$ sia alla letteratura di viaggio (oltreché a quella mistica). Leggiamone qualche esempio: "Io vidi un orbo che guardava un muto, / che predicava in mare a la campagna» (III I-2), "Io vidi un luco uscir d'una pantiera / a mezo zorno che lucia la luna, I tal ch'io non vidi mai in parte alcuna / si destro bo da pescar a rivera» (VII I-4), "Io vidi un armaiol dentro a Millano, / che avia imparata l'arte in Mongibello, / non operar ancudine o martello, / né avia altri mantici che l'ano» (XXXIV I-4), "Io vidi un gingator di bagatelle / gingar per certo si pullito e netto / che chi l'avesse visto avria ben detto: / 'El fa per certo cosse molto belle!'》 (ХХХVI I-4), o «Io vidi in deto al re Karlo un anello / lavorato a Parisi con grande arte, / ove 'I mondo intagliato era in tre parte, / la terra e il mare et ogni fumicello» (XLII I-4). Scrive al proposito Giorgio Raimondo Cardona: "Tensione continua nei testi di viaggio è l'insistenza sul valore di verità di quanto viene affermato; è come se la presupposizione costante fosse che quanto si dice non può essere vero perché è diverso, perché non risulta per comune opinione, perché chi parla è l'unica fonte. Di qui una particolare enfasi sulle formule asseverative: è come vi dico, lo so perché l'ho visto vi dico, lo so perché l'ho visto, ecc.». ${ }^{30} \mathrm{Nel}$ settenario dei sonetti caudati di Baldassarre compare il refrain "Questa non è busia», per tentare di persuadere il lettore della verità delle scenette improbabili. ${ }^{3 \mathrm{I}}$ E tuttavia non ci si dovrà lasciare ingannare dall'apparente gusto per l'invenzione smodata e per il fantastico: $i$ versi recuperano la tradizione, si diceva, dei mirabilia, talvolta abilmente occultati o manipolati. Per l'esegeta, il rischio di considerare inventate le immagini è sempre dietro l'angolo.

Fin dai primi sonetti è chiara la percezione di un riuso, stravolto, delle visiones medievali, come nel caso dei due versi «El si trovò già el Bivilaqua in mare / ad una terra murata di fuoco» (II I-2), che ricorda Visio Pauli, 9: "Et vidit muros igneos elevantes se ex utraque 
parte». ${ }^{32}$ Per attribuire alle visioni un carattere di straordinarietà, Baldassare fa un impiego disinvolto dell'iperbole, figura retorica che, non a caso, "consiste nell'oltrepassare la verita». ${ }^{33}$ Il sette, numero iperbolico di ascendenza scritturale (Prov 24, I6 e Mt I8, 2I) e presente abbondantemente nella Navigatio Sancti Brendani, ${ }^{34}$ è disseminato, talvolta come riempitivo, nella maggior parte dei sonetti. Baldassarre scrive persino di una prostituta-capra che, dopo svariati accoppiamenti, mette al mondo un figlio con sette teste: episodio che, pur sensibile ad un immaginario di gusto apocalittico (Ap I3, I) e al contempo leggibile come una punizione per una condotta immorale, ${ }^{35}$ risente anche del fascino esercitato da certe figure mitologiche mostruose (basti pensare che sette teste, di serpente, caratterizzavano Orto, il cane di Gerione). ${ }^{36}$ L'altro numero iperbolico, per esprimere una quantità indeterminata, ’̀ il mille, di origine latina, anche in questo caso largamente attestato (II 6 e I3; III IO e I7; IV 2 e I3; VI 6; VIII 8; XI II; XII IO; XX I7; XXIV I6; XXV 3 e I7; XXVIII 9, IO e I3; XXXVII I0; XLIV I7; LIV 3 e 17$)$. Esseri visibilmente iperbolici sono $i$ giganti, catalogati fra $i$ monstra e menzionati in IX I-2; XV I7; XXIV I6-I7; 37 il fabbro milanese del sonetto XXXIV, tratteggiato sulla base di un topos ben consolidato, ${ }^{38}$ oppure il facchino in grado di trasportare sulle proprie spalle torri ed edifici (LI); non manca un falcone dotato di una forza che gli permette di sollevare $i$ buoi legati ad un carro con tutto il suo contenuto (XVIII), ${ }^{39}$ simile a quello sparviere che riesce a rapire una nave carica di linci (XXXI I2-I4). ${ }^{40}$ Condividono poi un certa parentela il ragno che con la ragnatela riesce a catturare migliaia di animali (XXVIII) $e$ il pescatore che con la rete si procura settemila cammelli $(X X X V)$.

L'iperbole conosce varie declinazioni. "Quando il re Karlo conquistò la Spagna, / prima che fusse morto, il conte Orlando, / una mula spagnola cavalcando, / vene in un di da Napoli in Lamagna» (X I-4): abbiamo a che fare con uno spostamento compiuto in un lasso di tempo brevissimo, situazione che ricorda da vicino il viaggio magico narrato da Boccaccio, Decameron, X 9, 70: «Il Saladino a un suo nigromante, la cui arte già espermentata aveva, impose che egli vedesse via come messer Torello sopra un letto in una notte fosse portato a Pavia». ${ }^{4 \mathrm{I}}$ A questo si aggiunga che il motivo sembrerebbe di origine orientale: si diceva che il cammello volante fosse in grado di coprire un miglio in un istante. ${ }^{42}$ Altri riferimenti alle ampie distanze si affacciano in $V I I$, dove una 
torta, da Milano, «in un bocon [ciascuno] la manda in Tartaria», o in XXV 8: «e bianco saffaran per quelle cave / coglievan per portarlo fino in Spagna», richiamo, anch'esso, dal sapore topico, come testimonia Giacomino da Verona, De Babilonia civitate infernali, v. I0z: «esro scovai de spine da Roma enfin en Spagna». ${ }^{43}$

$N$ el Bosadrello gli adynata sono esibiti con una certa generosità: «A megia nocte, poi nel far del giorno» (IV 9), "a mezo zorno che lucia la luna» (VI 2), e «danzar nell'alba che 'l sol tramontava» (XI 2) sono endecasillabi che risentono di soluzioni burchiellesche (I sonetti del Burchiello, XCIX I: "A meza notte, quasi in sulla nona», e ivi: СХХХИІІ 2: "di meza nona in sul levar la stella»), ${ }^{44}$ ma presentano una consonanza con taluni fenomeni prodigiosi descritti nei testi antichi, uno per tutti IV Esdra 5, 4-s. Prodigioso o, se vogliamo, favolistico, è il caso dell'immagine "Una volta la luna venne in terra» (VI I), che conosce precedenti nella letteratura latina, tra $i$ quali Seneca, Hercules Oetaeus, 467-68: «[...] carmine in terras mago / descendat astris Luna desertis licet». ${ }^{45}$

Come anticipato, versi che narravano di singolari e comiche metamorfosi potevano essere recuperati nei sonetti burchielleschi: «a Moncia [Monza] se ne fé si fatta pieta / che la corona si copri di ruggine / e la gallina diventò testuggine, / che fé trassecolare ogni profeta» (I sonetti del Burchiello, XLII 5-8), tuttavia l'attenzione di Baldassarre si concentra anche su incroci o parti bizzarri: in un sonetto si parla di una gallina che rimane incinta di un gatto (XVII IG), mentre in un altro di una genesi improbabile a opera di due furfanti che litigavano per una torta: "Questa non fu busia: / se una volta qui del ver mi apago, / nel terzo mese ognun parturi un drago» (V I5-17). La chiusa richiama, oltre alla genesi del drago (Ap I2, I-3), i parti mostruosi, ben descritti, ad esempio, nelle Fatrasies d'Arras, I9, I: "Dragons de geline» (ovvero "Un drago nato da gallina»), ${ }^{46}$ la cui fonte è probabilmente Gautier de Châtillon, Alexandreis, X 343-44: "De celo ueri lapides cecidere. locutus / Agnus in Egypto est. peperit gallina draconem», ${ }^{47}$ travasato anche nel De Amore di Andrea Cappellano: «et peperit mitis gallina draconem>. ${ }^{48}$

Apparentemente mostruosa è la gallina descritta in XVII I-4: «El naque una gallina in Padüana / che avia un corno longo ben due miglia / e non ti parà questo maraviglia: / ancora avia in testa una fontana». Baldassarre, nel primo verso, introduce la "gallina padovana", in 
genere nota per le sue dimensioni ragguardevoli e «caratterizzata da un abbondante ciuffo di penne in testas (Zingarelli), qualità che viene fatta incrociare con la gallina d'India, che presentava in testa una sorta di corno, almeno secondo una testimonianza dei primi del Seicento. ${ }^{49}$ Alcuni dei testi della nostra raccolta si soffermano su piante dalle capacità straordinarie, come nel sonetto XXIX:

Eli era in la pigneta di Ravenna un pin molto magior che gli altri assai che facì cossa non veduta mai, se noti ben le lettre di mia penna.

Di quel non si facìa nave ni antenna [pennone], come qui apertamente vederai, ma daseva ad Italia magior' guai ['danni'] che non fa a la Cecilia monte Ethenna. [Etna]

Nel tempo che gli altri arbor' fano il fructo questo faceva cervi molto grandi che un omo armato a dosso tenìa.

Era il paese da quilli distructo: vivian di faxan', confecti e ghiande e corsegiavan tutta Lombardia.

Questa non fu busia, nasceva il cervo cum barde e sopravesta e l'omo avia la lanza e l'elmo in testa.

Il luogo dal quale Baldassarre avvia la descrizione è, letterariamente, di tutto rispetto: la pineta era stata ricordata da Dante, Purg. XXVIII I9-2I: «tal qual di ramo in ramo si raccoglie / per la pineta in su'l lito di Chiassi, / quand'Ëolo scilocco fuor discioglie», dove appariva quasi un luogo edenico; ${ }^{\circ \circ}$ in una pineta dei pressi di Ravenna, poi, è ambientata la novella di Nastagio degli Onesti (Decameron, V 8), mentre, nello stesso luogo, Franco Sacchetti colloca avvenimenti bizzarri (Trecentonovelle, $C I V$ б).

Nella prima terzina mi sembra che Baldassarre accenni a una genesi piuttosto insolita: più che a un nomo a cavallo di un cervo, penserei a un essere ibrido, metà nomo metà animale, tipologia cara alla cultura medievale, e soprattutto alla letteratura di viaggio. ${ }^{\text {I }}$ Un recupero, direi, del motivo dei centauri, nei quali la parte animale, costituita dal cervo, risulta attestata già nell'arte etrusca: ${ }^{2}$ Baldassarre avrà di certo avuto a mente la figura del sagittario. ${ }^{53}$ La natura ibrida degli esseri riceve- 
rebbe una conferma dai cibi consumati: se fagiani e dolci si addicono agli uomini, le ghiande di certo di più agli animali.

Di piante dalle proprietà simili a quella sopra descritta abbiamo una testimonianza più tarda:

produce arbori che fruttifican meloni, de grandezza d'una botte, ognuno nutrimento buono, come da noi si dà del pane. Ma delle seme ascolta maraviglia, le ponevano in acqua che li radiava il sole, et per spatio de una revolution lunare, sgionfavano fin che non possendo più la scorza estendere se rompea, della quale sorgevan arditi polledri, et passato l'anno eran apreciati corsieri. ${ }^{54}$

Simile al sonetto appena visto è il seguente, nel quale si racconta di un albero che produce sirene musicanti (XXVI):

Un arbor nasce nel septentrione che da megio genar fa fior' d'argento e quando il fructo vèn a compimento nascon sirene cantando cantione.

E ciascuna cavalca un gran lëone, organ sonando over altro instrumento et ogni fior ne fa ben cinquecento, sì che per certo è grande amiratione.

Qui danzan gli lëon' tra fiori e fronde e qual di loro a ben cantar s'è dato, che ad ogni gran signor ben piaceria, quando vien l'ora poi che 'l sol si asconde et ciascaduno al suo fior è tornato, in trïumphanti leti si copria.

Questa non fu busia, di panno d'oro è ogni copritura e fu di perle una gentil figura.

Il sonetto si apre con un'immagine da flora paradisiaca, che sembrerebbe essere rimasta impigliata nel rampino di Marino (Adone, X 38, I-2: "Ha di diamante la radice e'l fusto, / di smeraldo le fronde, i fior d'argento»), per poi incentrarsi sul frutto straordinario, sirene musicanti, per l'appunto. ${ }^{5}$ Benché nelle arti figurative medievali le sirene potessero essere accostate ai leoni, è la tradizione classica che conosce la raffigurazione della dea Cibele (o Demetra) in groppa a un 
leone. Inoltre, la descrizione dei letti particolarmente sfarzosi sembrerebbe riproporre un motivo noto, di cui un esempio affiora nel Voyage de Charlemagne, XXII 4I9-32:

Li reis Hugun li forz Carlemain apelat, Lui et les duzce pers si.s trait a une part;

Le rei tint par la main, en sa cambre.l menat,

Voltue, peinte a flurs, a peres de cristal;

Une escarbuncle i luist e cler reflambeat, Confite en une estache del tens le rei Golias.

Duze liz i ad dous de quivre et de metal, Oreillers de velus e linçous de cendal;

$\mathrm{Al}$ menur < unt $>$ a traire vint beof et quatre car.

Li trezimes en mi est taillez a cumpas,

Li pecul sunt d'argent et l'espunde d'esmal.

Li cuverturs fud bons que Maheuz uvré at, Une fee mult gente que le rei <le> dunat;

Melz en vaut li conreiz del tresor l'amiral. ${ }^{56}$

Coerentemente con l'aspetto iperbolico che attraversa l'intera raccolta, $i$ frutti degli alberi presenti nel Bosadrello sono giganteschi. Si prenda il caso del sonetto XXVII:

Volendo campegiare el Tamberlano per aquistar la Franza e la Borgogna, prese il camino verso di Sansogna, per fugir le montagne e gir per piano.

Et, per non amalar ma viver sano, alozò el primo dì ne la Guascogna; non creder ch'io ti dica qui menzogna: el dì sequente fu gionto a Milano.

Trovòvi un piè di verze tanto grande che sotto gli alozò la fantaria d'un milion e mezo di persone, e d'una foglia sol si fé vivande con carne di vitelli e di castrone, che cenò ben tutta la compagnia.

Questa non fu busia, e' gli era un piè di verze zenovese che facìa ombra fino in Bolognese. 
Leprime due quartine presentano di nuovo il motivo delle distanze coperte in tempo rapidissimo, sul quale non ci soffermeremo. Al cavolo genovese ( $v$. Ib) accenna Giulio Cesare Croce nell'Indice universale della libraria; ${ }^{57}$ quel che però attira maggiormente il nostro interesse è la descrizione della pianta gigantesca-purpresente tra $i$ Detti di Poliziano (XCIII) -, che sembraprovenire dalla letteratura dei viaggiparadisiaci:

L'arbero del Paradiso è facto a questo modo, cioè el suo pedale diventa grosso et grande e lle soe foglie sono asimigliante ad una lingua e sono sì large che quatre o sei persone gli starano a giacere suso una de quelle foglie ed è longa quanto una lancia. ${ }^{58}$

Le caratteristiche delle piante e degli arbusti illustrati appartengono alla tipologia contemplata nel Paradiso terrestre, luogo richiamato in maniera esplicita nella prima quartina del penultimo sonetto della raccolta: «Io fui pur una volta in Paradiso, / ché mi condusse l'angel Gabrïle, / là dove io vidi cose tanto belle / che non è omo che non avesse riso» (LVI I-4). ${ }^{59}$ Qui spunta un'altra pianta straordinaria: "Tra l'altre cosse ch'io vidi mirande, / arbori grandi cum le gambe d'oro» (LVI 9-IO), per la quale basti pensare a Dante, Purg. XXIX 43-45: «Poco più oltre, sette alberi d'oro / falsava nel parere il lungo tratto / del mezzo ch'era ancor tra noi e loro». Il motivo affonda nella tradizione orientale ${ }^{60}$ e conosce esempi nella letteratura mistica: «Allora lui mi disse e manifestò una cosa molto stupenda, cioè che, dopo molte cose che li furono mostrate dello stato delle Chiesa militante, vide una arbore molto bella e alta, la cui radice era d'oro e il pedale d'argento, le foglie d'argento innorato» ${ }^{61}$ (si veda pure Boccaccio, Filocolo, II 32 s: "E sopra tutte queste cose v'era intagliata la imagine di Giove, vestita di più ricca roba che quella che Dionisio fero già gli spoglio, intorniato d'alberi d'oro, le cui frondi non temevano l'autunno, e i loro pomi erano pietre lucentissime e di gran valore»), ${ }^{62}$ con attestazioni più tarde: $« L i$ meloni et li arbori sono in colore d'oro, lustri et trasparenti da ogni parte, hanno grandezza gli arbori de amplo et sparso platano». ${ }^{63}$ In modo simile, nel sonetto XXXII 9-I0, si parla di un giardino che "Rape et ravanei facia d'argento, / carotte e porri di pietre preciose», motivo parzialmente riconducibile a quello delle piante che producono gemme, anch'esso conosciuto in Oriente. ${ }^{64}$ I versi appena citati vanno accostati a quelli della prodigiosa semina registrata nell' antica Pechino: 
"Io vidi in Camalech un ortolano / che seminava le pietre pretiose» (XLVIII I-2). ${ }^{65} \mathrm{Nel}$ commento al passo erano stati addotti come riscontro alcuni versi, quasi coevi, di Felice Feliciano, nei quali si parla di una semina di rubini, tuttavia una testimonianza più tarda (I532), ma che probabilmente attinge a una fonte comune, racconta di terre lontane dove alcuni giganti compiono una simile operazione: «Sono questi [scil. i Giganti dell'isola Nominanza] della terra mirabili cultori et come tra noi del grano, meglio, fave, pizoli et simigliante seme fanno gli villani, non altramente essi le monete d'oro et d'argento, le perle e ogni gemma pretiosa seminano, cultivano et finalmente recoglieno et conservano in le fosse, case et magazenì. ${ }^{66}$

Tra i protagonisti più evidenti dei versi appaiono gli animali, prelevati da un ampio bestiario - in linea fedele con l'esplosiva poesia burchiellesca-, e ritratti di frequente in azioni improprie, con lo scopo di offrire la visione di un mondo rovesciato. Notoriamente le cicogne sono animali miti e bollati come vili (si veda Philippe de Thaün, Bestiaire, v. 2635: "Vil oisel est ciguine»), ${ }^{67}$ mentre Baldassarre le ritrae battagliere: «Ben si provaro alora le cigogne / con lor corsier' pogliesi covertati, / che chi nol vide mai nol crederia» (IX 9-II). ${ }^{68}$ Un secondo caso: seppur richiamate in pose belliche nella Batracomiomachia, sembra poco probabile che le rane possano inseguire minacciose Alessandro Magno: «Le rane alora fessero soggiorno: / cavalcâr tutte su mille lëoni / e per trovarlo gîr in Picardia» (IV I2-I4). Peraltro, anche in questo caso incontriamo $i$ leoni cavalcati, ma l'associazione tra batraci e felini è piuttosto antica e consolidata. ${ }^{69}$ Altrettanto improbabile che un grillo ferisca a morte il forzuto giudice biblico Sansone (VIII), che il re Pipino venga ucciso da un insignificante legume come il lupino (X I6-I7), che una lumaca sconfigga e uccida Ettore (XV 9-II), ${ }^{70}$ o che un calabrone riesca ad abbattere sei giganti (XV I6-I7). Un ulteriore caso è rappresentato dai tre versi: «et poi una oca d'un gran catelano, / armata di corrazza e bone arnesi, / pigliar tutti i lëon' di Barbaria» (III I2-I4). ${ }^{71}$ Il rovesciamento dei ruoli contempla altri casi: $i$ tre conigli che catturano settemila volpi (VII $5-6)^{72}$ o le formiche che prevalgono sui grifoni (XIII). Le modalità applicate sono relative al sovvertimento dei ruoli oppure alla palese incongruità: valga l'esempio del gatto che agguanta un delfino, anziché un pesce di taglia minore ("et una gatta nel mar Occeàno / aver preso un dolphin de mille pesi》, III 9-II), all'asino che cattura le pernici (III I5-I7), ${ }^{73} \mathrm{e}$ all'elefante e all'orso rapidi e agili nel ballo (XI 9-I2). 
Privi dell'aspetto bellicoso, altri animali contribuiscono a formare un contesto irreale: valga il caso dei maiali (animali noti per essere l'emblema dell'accidia), che intraprendono la carriera di mercanti, fino a quando uno di loro verrà incoronato re: "Questa non fu busia: / come Livio in più parte ne rasona, / l'un di loro si fé re di corona») (XII I5-I7) ${ }^{74}$ o della cerva dotata di artigli (VII Iठ). Più complessa l'immagine di ХVI I6: «che già mel disse a Londra un bianco corbo» (e cfr. «un cigno niero» di XXИIII IO). ${ }^{75}$ In principio, si osserverà che la collocazione del volatile non è peregrina: $i$ corvi sono legati all'Inghilterra da varie leggende $;^{76}$ inoltre, corvi parlanti erano attestati in Oriente: $« E$ stan< $>0$ con questi frati, et me contorreno che in quella terra era uno giocularo che era molto riccho e, fra l'altre sue maraviglie, lui havía uno corbo che favellava de ogni lenguaggios. ${ }^{77}$ Il colore è si paradossale, ma non andrà trascurato che nella tradizione dei bestiari l'uccello nasceva bianco: "Nasce ogni corbo, per natura, bianco / e pascesi dal cel del dolce manna». ${ }^{78}$

Un'immagine dall'evidente significato paradossale si incontra nel secondo sonetto, dove si descrive una «vaca volare» (II s), ${ }^{79}$ da rapportare al verso «due buffale cum alle bianche e belle» (VI IØ). Ancora nel sonetto II una civetta è impegnata nel suonare uno strumento musicale: "Questa non fu busia: / poi vide in Babilonia una civeta / che molto ben sonava una trombetta》 (II I5-I7). Secondo la tradizione biblica, la civetta è presente a Babilonia assieme ad altri animali impuri (Isaia I3, 2I: «sed accubabunt ibi bestiae, et replebunt domus eorum ululae, et habitabunt ibi struthiones, et pilosi saltabunt ibi»), ${ }^{80}$ ma l'animale musicante è di per sé una rappresentazione comica, che spesso trova posto nel mundus inversus: ${ }^{8 \mathrm{I}}$ se ne possono vedere altri esempi: «Io vidi un orbo che guardava un muto, / che predicava in mare a la campagna, / dicendo aver veduto già in Ispagna / una formica sonar un lëuto» (III I-4) (12 $^{8}$ "ret era quivi un asin che sonava / una arpa grande cum la destra mano» (XI 3-4), quest'ultimo caso ben riconoscibile. ${ }^{83}$ Altrettanto comica la scena dei buoi con gli stivali ( Nel mar di Grecia, presso a la Valona, I nascono bovi cum calze e stivali, / cinti cum archi e forniti di strali, / legieri e destri de la lor persona», XXXVII I-4), associabili alla "bertuccia in zoccoli". ${ }^{84}$

I motivi racchiusi nel Bosadrello si intrecciano insistentemente. Già nella Navigatio Sancti Brendani (IX) si parlava dellisola delle pecore giganti: ${ }^{85}$ tra $i$ nostri versi si incontra un volatile dalle dimensioni 
impareggiabili: «un struzzo tanto grande et tanto altano, / che fra le gambe tutto Milan tenia / e la testa giongia al meregiano. / E quando l'era tempo ch'el piovia, / quel ch'io ti parlo non til dico in vano, / tutto Millan cum una ala coprià (XLIV 9-I4). Qualcosa di affine è presente nel Libro della Scala (XXIX), a proposito del gallo:

Postquam ego Machometus vidi que supra narrata sunt, Gabriel et Raphael ostenderunt michi quemdam gallum, qui proprie Dei erat. Hic namque gallus ita magnus erat quod ipse tenebat caput suum et crestam ad celum, ubi Deus est et cathedra ejus; et pedes suos ad magis infimum terre septime, de qua in sequentibus audietis. Et quid vobis recitarem de forma ipsius? Deus fecit eum sicut placuit sibi ipsi. Erat enim gallus iste unus ex angelis Dei qui, licet ita fuerit, non tamen sciebat ubi Deus esset. Hoc autem faciebat quod ipse semper Deum laudabat et in suis dicebat laudibus: 'Benedictus sis tu, Domine Deus, ubicumque sis'. Habebat eciam gallus iste alas que ita magne erant quod, cum aperiebat eas, penetrabat cum ipsis omnes celos omnesque terras que sunt ab oriente usque ad occidentem. ${ }^{86}$

Passo che si allaccia, a sua volta, a quella di un altro sonetto, il XI:

Io mi ricordo aver vedù dui galli, che l'un di loro stava in orïente e l'altro ne le fine d'occidente, cum penne cremosine ch'eran gialli.

Avian le creste lor di chiar' cristalli et era ognun de voce sì possente che, s'el cantava quel ch'era in Ponente, che avia li piedi tutti di coralli,

quel de Levante attento lo ascoltava; e quel cantava ogni or greco e latino, l'altro unde nasce il sol gli rispondia.

A far bataglia un dì si diffidava e ciascadun di lor entrò in camino e lì su nel mar Rosso sen venìa.

Questa non fu busia: sopra quelle aque s'ebbero' amazare e però è che gli è rosso quel mare.

Senza dover scomodare testi di mistica, ci si potrebbe accontentare della presenza di galli giganti in Oriente: «Et Eartendome de questa terra io 
venni verso oriente a una città che è sopra al mare, che largamente gira più de trenta miglia et è chiamata Foggia: e sonno li magiori galli del mondo e le galline sonno tutte bianche como neve»). ${ }^{87}$ Né va dimenticato che nelle terre orientali erano diffusi i combattimenti tra galli, come mi pare indirettamente evocato nel sonetto. ${ }^{88}$

Oltre al brulicante mondo animale e vegetale, $i$ testi vengono costellati di frequenti richiami a opere architettoniche, oggetti e costruqioni strabilianti. ${ }^{89}$ Si prenda il sonetto XXX:

El fece Codro già in mare un ponte che tutto di perle era lavorato, di adamantina calzina murato, che da Savoia giongeva in Piemonte.

In questo Cesar poi fu facto conte e da lui fu di corali merlato e fecegli una torre d'ogni lato che passava d'alteza ogni gran monte.

Ognuna avia settemilia campane de fino argento, e 'l martello era d'oro, che da Ponente a Levante se udia.

Venne per caso che l'aquistò el Gran Cane: gittò per terra quel nobil lavoro e settemilia cità fece in Turchia.

Questa non fu busia, el ne fé primamente Antrinopoli et Pera bella con Constantinopoli.

Dopo l'evidente iperbole del v. 4, i versi indugiano su un ponte sfarzoso costituito da materiale pregiato. Nella tradizione della letteratura odeporica (a partire dalla Navigatio Sancti Brendani) si parla sia di un ponte d'oro sorretto da quattro travi di cristallo, granato, perle e topazio, $9^{\circ}$ sia di un grande ponte nel Catai: $9^{\mathrm{I}}$ "E sopra questo lago [scil. nei pressi di Ghambalai] viddi io el più bel ponte che credo che in tutto el mondo se possesse trovare el simile, de milli ragioni de marmori e con tanti intagli che è uno stupore a ddire». ${ }^{9^{2}}$ Inoltre, un castello orientale, costruito con pietre preziose e metalli pregiatissimi, secondo l'immaginario della Gerusalemme celeste, è al centro del sonetto XXI. ${ }^{93}$ Lusso e sfarzo orientali vengono contemplati nel sonetto $\mathrm{LV}$, dedicato al Prete Gianni, noto per le sue ricchezze: 
Ogni anno fa una festa il Prete Ianni, settemillia carette d'òr fa armare e fàlle corer un palio per mare facto cum gran fatica e molti affanni.

Prima ch'el se fornisca, sta sette anni settemillia maestri a lavorare et altri tanti sonci a ricamare lavor' di drapo d'òr, non d'altri panni.

Settemillia elephanti per careta sono a tirarla poi quando si corre e settemille miglia è quella via.

E córon più velozi che saetta: non si porian queste cose disporre chi non vedesse la lor maestria.

Questa non fu busia, ogni caretta ha settemillia donne che ognuna sta d'òr fin fra due collonne.

La festa del primo verso potrebbe richiamare le quattro feste annuali tenute dal Gran Can, ricordate da Odorico da Pordenone e John Mandeville. ${ }^{94}$ Anche la descrizione successiva sembra non discostarsi molto da quella del carro del Gran Can:

E quando lui [scil. il Gran Can] volesse andare da una terra a l'altra, el monta in un bellissimo carro de legno; et alhora questo carro è ornato de tanti drappi d'oro e de prete pretiose e perle grosse che per certo era uno stupore a vedere. E questo carro era menato da quatro elephanti coperti triumphalmente; e sopra ad questo carro $<_{\mathrm{v}}>\mathrm{a} \times \mathrm{x}$ girfalchi, e quando vanno per via vanno sempre ucellando.95

Infine, i drappi d'oro (cfr. v. 8) sono più volte citati dal Mandeville. ${ }^{96}$ A creare un'atmosfera meravigliosa contribuiscono anche oggetti dai poteri straordinari, come una fontana e uno specchio (XLIII):

Io vidi in la gran corte al Saladino una fontana grande di òr potabile, che a veder era cosa mirabile, piantata e posta in un suo bel giardino;

et poi di quella usciva un fiumecino molto ben grosso, assai corent'e amabile. 
Deh, odi s'el facìa cossa notabile, che d'un om vechio el refacìa fantino,

et se gli era fantino il facea vechio e poi il ritornava al primo stato, sì che chi ne bevìa mai non morìa.

E sopra la fontana era un spechio da settemillia stelle circundato e dentro a questo ogni ora si vedìa, questa non fu busia, quel che i cieli dispongon de la terra e quando serà pace et quando guerra.

Il sonetto si apre con il richiamo al fons iuventutis, ${ }^{97}$ per concludersi con un riferimento patente alla catoptromanzia: tra $i$ tanti esempi adducibili di questa forma divinatoria, studiata da Armand Delatte, ${ }^{8}$ è interessante osservare che nel medioevo si riteneva che Virgilio fosse l'inventore di uno specchio magico dalle strabilianti proprietà, narrate nel Roman des sept sages: ${ }^{9}$

Virgilles fist un mireor,

Ki molt par fu de grant valor.

Molt fu cil mireours prisiés;

De hauteche avoit bien cent piés

(vv. 3969-82)

Molt estoit em pais cele terre,

De nule part n'i avoit guerre.

Au mireor courent savoir

Quant nule guerre i doit avoir,

Mais hom ne l'osoit envaïr,

Rois ne dus ne quens assaillir

(vv. 3995-4000).

Un intero sonetto viene dedicato a un mago e alle sue capacità: nella coda si legge "Questa non fu busia: / ch'el ti facia venir del naso un grugno, / andar a torno col falcon in pugno» (XXXVI I5-I7). Sembra che la trasformazione del naso in un grugno suino ricordi, almeno parzialmente, la metamorfosi patita dai compagni di Ulisse (Od., X, I87-466). In più, nel medioevo si credeva si potesse far mutare un essere umano in maiale grazie ad un unguento magico, come è chiarito nel Liber Ane- 
guemis, 28 (Capitulum unguentorum): «Si volueris convertere formam hominis ad formam symij aut porcijy), ${ }^{100}$ senza tralasciare che nella parte centrale del Trittico delle tentazioni di Bosch (Lisboa, Museo Nacional de arte antiga) si può osservare una nomo dal palese volto suino. ${ }^{\text {Ior }}$

Una delle numerose spie che rimandano all'Oriente si affaccia in XXIV I-8:

Ne l'India Mazore, verso di Ethiopia, di balsamo si vede un alto mare, che non si può cum legni navicare, tanto de lëompardi gli è gran copia.

Et quel paese ha d'aqua tanta inopia che cozeno nel balsamo il manzare et fanvi el lor bestiame abeverare come de lor scripture in ver s'acopia.

Anche in questi versi Baldassarre racconta di una scena verosimile, pur con tratti irreali: in Oriente era collocato l'albero del balsamo, ${ }^{\mathrm{IO}}$ fumi di balsamo appartengono alla tradizione ebraica ${ }^{\mathrm{IO}}$ e scorrono nelle visioni cristiane. ${ }^{\mathrm{I}}{ }^{4}$ Del motivo si trovano tracce anche in poesia: mi riferisco ai versi di Lapo Gianni: "Amor, eo chero mia donna in domino, / l'Arno balsamo fino» ${ }^{\text {10 }}$ e all'incipit angiolieresco Se tutta l'acqua balsamo tornasse. Nei sonetti sono sparsi altri elementi di origine orientale: animali specifici come $i$ cammelli (XXXV I 6$),{ }^{106}$ e $i$ pappagalli (XXI I3; XXVIII I0), ${ }^{107}$ piante come l'aloe (XXXVI I0), ${ }^{108} 0$ spezie come il pepe (xXV 6). ${ }^{\text {Iog }}$

Si è accennato in principio alla fortuna riscossa dal Bosadrello nel Cinquecento fino al Seicento. I versi di Baldassarre non costituiscono certo un caso isolato nella letteratura nostrana: testi affini possono essere recuperati senza difficoltà nel Cinquecento. Penso in particolare ai versi del Mariazzo molto piacevole et da ridere di donna Rada bratessa: stampato nouamente (s.n.t. [ma tra il $I 520$ e il I525]), ${ }^{\text {IO }}$ del quale Mario Chiesa ha offerto un'ampia trascrizione, che qui riproponiamo in parte:

Un mazo de carotte si corsono con furia rovinando una anguria in un fasso de stopa. 


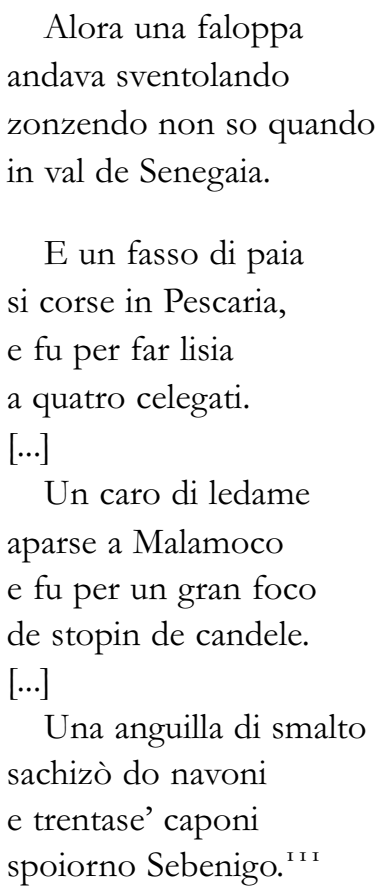

Soluzioni più strettamente vicine a quelle del Bosadrello si possono ricavare dalla Farza de li massare del poeta napoletano Velardiniello (XVI sec.), come rilevò tempo addietro Luigi Emery: ${ }^{\mathrm{II} 2}$

Io vidde un dì iocare a paro e separa

No riccio, no salusa e na cecogna;

E na ciaula cantare co no cefaro,

No sorece sonare na zampogna,

No grancio a petra in sino co no leparo

No gallo traiettare a la cedogna,

$\mathrm{Na}$ vorpe predicare sopra n'ammendola,

E po vocare con saglipendola.

Io vidde questo, e so che non fui uorbo,

$\mathrm{Na}$ scigna che frieva certe sarache;

No gammaro a llottare co no cuorbo,

E fare sette piezze de lle brache;

No castrato iontrar sopra no suorbo,

E ficcarese nel cul doie pastenache;

Io vidde questo, e fo de carnevale,

Tre anatre a danzar co lle stivale 
Io vidde un gran miraculo a la Costa:

Un ascio cavalcare un orso imbardella, $E$ vidde con quest'occhi una ragosta Far la pavana a suon de ciaramella; E no vozzacchio farese na sopposta, E po iocare a mammara cecella; Po vidde dar no iuntro a na cestunia, $\mathrm{E}$ dare a na marotta vinte punie.

Ho visto no palumbo gorgiuluto

Sbattere l'ale e deventare luccaro:

E no reviezzo fare no sternuto, Ad Ottaiano e po sentirse a Cuccaro; Ho visto ancora un passaro acceputo, Mangiare sorva e po cacare zuccaro; E na cornacchia far na capetommola, N'oca co na maruzza a spaccastrombola.

Ho visto no galluccio pataniello,

Pelarese co lo pizzo le parpetole;

E n'aseno menar co no cortiello,

$\mathrm{E}$ accidere a na fico tre focetole;

No polece strengato a in ioppariello

Scrivere co na penna certe cetole;

Poi vidde armarse in bianco na formica, E co na lanza secutar una pica. ${ }^{13}$

Esiste una continuità del genere poetico, come testimonia l'opuscolo popolare, già segnalato da Rossi, Strambottoli venuti di qua, di là, di su, di giù, e da diverse parti del Mondo [...] (Firenze, Salani, I878). ${ }^{\text {I } 4} \mathrm{Ne}$ riporto un paio:

Stanotte, a mezzanotte ho visto il sole

Sotto di un'ombra a grattarsi la rogna, $\mathrm{E}$ un lupo che friggeva due braciole

Nell'acqua fresca senza vergogna;

Un albero che avea cento figliole

Le son morte agli studi in Catalogna;

Di più sentite: un Asin con le corna

E' porto vià la scuffia di una Donna. ${ }^{\text {Is }}$ 
Oppure:

\author{
Sopra un albero un pesce vidi un giorno \\ Che ben cantava allo spuntar del sole, \\ E molta gente ad esso erano attorno \\ Per sentir l'esclamio di tai parole: \\ «Lo star nell'acqua non m'importa un corno \\ Se la mia amante alfin più non mi vuole!» \\ E mentre che facea questi lamenti \\ Ingoiò un porto, e mille bastimenti. ${ }^{16}$
}

Gli esempi potrebbero proseguire, soprattutto per mostrare talune affinità specifiche con i versi di Baldassarre: "Correvan per le poste due lumache / Sopra la scorza di una grossa arancia» (25, I-2) e «Una corrente lumaga non tarda» (Bosadrello, XV 9); "Montò a caval d'una montagna un'occa / Sfidando ai pugni un orso barbaresco» (I4, I-2) ed «et poi una oca d'un gran catelano, / armata di corrazza e bone arnesi, / pigliar tutti i lëon' di Barbaria» (Bosadrello, III I2-I4); o "E un gallo che veniva dall'Oriente» $(22,3) e$ "Io mi ricordo aver vedù dui galli, / che l'un di loro stava in oriente» (Bosadrello, XL I-2). Si pensi poi a certe architetture miste: "Sopra la chioma di un uccel che vola / Si fabbrica un castel con una torre» $(8, I-2)$ ed «El naque una gallina in Padïana / che avia un corno longo ben due miglia / e non ti parà questo maraviglia: / ancora avia in testa una fontana» (Bosadrello, XVII I-4); «E finalmente un campanil di vetro» $(I I, 7)$ e «un campanil tutto di chiar cristallo» (Bosadrello, XLV 9). ${ }^{17} 7$ Tuttavia un aspetto di un certo rilievo va segnalato a proposito di questo curioso opuscolo: almeno tre di questi strambottoli, e più precisamente $i$ numeri II (Nacque la guerra delle rane antiche), I4 (Montò a caval d'una montagna un'occa) e I6 (Era di notte e non ci si vedea), erano già inclusi nel Poeta fanatico di Goldoni, risalente al $1750 .{ }^{\text {I }} 8$ Ad attingere a questa tradizione, ravvivandola, è stato Gianni Rodari, il quale, anche attraverso la mediazione di Lear e del Surrealismo francese, ha riproposto $i$ motivi delle poesie della menzogna e delle "canzoni alla rovescia" in tutt'altro contesto, le favole e le filastrocche per bambini. Proprio nei primi quattro versi di una poesia intitolata Canzone alla rovescia, un nonno, rivolgendosi al nipote, racconta: "Conosco una canzone alla rovescia / e alla rovescia la voglio cantare: / una foca volò sul Monte Bianco / e una giraffa camminò per mare», 
e più avanti: "Conosco una canzone alla rovescia / e alla rovescia ve la voglio dire: / ho visto seminare sulle nuvole / e sulle nuvole il fiore fiorire»). ${ }^{\mathrm{II}} 9$

Meno mostri, certo, ma più meraviglie e paradossi: si ritorna, mutati pubblico e tempo, a stupire, con i racconti che solo $i$ viaggiatori sedentari sanno intrecciare.

Giuseppe Crimi 
I. Per le questioni più specifiche rimando alla mia Introduzione a BALDASSARRE DA Fossombrone, El Menzoniero overamente Bosadrello, a cura di G. Crimi, Casoria, Loffredo, 20ı; fin da ora, per le citazioni, farò riferimento a questa edizione. Ringrazio Franco Pignatti per la lettura del dattiloscritto.

2. Si veda, ad es., M. Vocino, Storia del costume. Venti secoli di vita italiana, Roma, La Libreria dello Stato, i 952 , p. 350.

3. S. Casali, Annali della tipografia veneziana di Francesco Marcolini, Forlì, Matteo Casali, г 86 г, p. г 22, n. I г: «Il Bugiardello di Luigi Brocardi. Venetia, per Mattio Pagan, I 558 , in 4. figur. in legno».

4. L'esemplare si trovava e si trova presso il Museo Correr di Venezia. Un altro è conservato nella Biblioteca della Fondazione Giorgio Cini di Venezia: cfr. C. Callegari, Scheda dell'ed. Lorenzo Spirito, Libro de la ventura, Venezia, Mattio Pagan, I557, in La vita nei libri: edizioni illustrate a stampa del Quattro e Cinquecento dalla Fondazione Giorgio Cini, catalogo a cura di M. Zorzi, Venezia, Edizioni della Laguna, 2003 , pp. 246-48 e D. RHODES, Catalogo del fondo librario antico della Fondazione Giorgio Cini, Firenze, Olschki, 201 I, p. 244 (numero 552). Si veda inoltre C. Callegari, Per Pietro Aretino iconografo e Andrea Schiavone silografo, in Gli affanni del collezionista. Studi di storia dell' arte in memoria di Feliciano Benvenuti, a cura di C. Callegari, Padova, Il Poligrafo, 2005, Pp. 95-I 32, a P. I 2 I.

5. Cito dall'esemplare della Fondazione Cini (ringrazio la dottoressa Lucia Sardo per la cortesia e la disponibilità).

6. Si veda Tomaso Garzoni, La piazza universale di tutte le professioni del mondo, VIII: De' formatori de' pronostici, tacuini, lunari e almanacchi: «Non vedi tu, meschino, che il circolo della piazza non è se non di gente che si fa beffe del fatto tuo? Chi chiama il tuo tacuino un bugiardello; chi nomina il tuo pronostico il pronostico del Gonella; chi lo guarda ridendo; chi lo legge smattando; chi lo canta come una lezione da pedante» (ed. a cura di P. Cherchi e B. Collina, 2 voll., Torino, Einaudi, i 996, vol. I, p. 2 I 4).

7. Nell'Oesterreichischer Catalog Vierter Theil Verzeichniss aller im Jahre 1862 in Oesterreich erschienen Bücher und Zeitschriften in italienischer Sprache, Wien, Verlag des Vereines der österreichischen Buchhändler, ı 863, p. 6, è registrato «Il Bugiardello, ossia l'oracolo degli antichi, che scherzando predice le sorti rispondendo esattamente ad ogni domanda. Giuoco dedicato al bel sesso o alle conversazioni nelle sere invernali, con una bussola ed una spiegazione del giuoco $2^{a}$ edizione 8 Trieste A. Levi [1 862]", ma nel «Giornale generale della bibliografia italiana», s. I, IV, is dicembre i 864, I 2, p. 90 è censita un'edizione Trieste, Coen; la prima edizione, del Levi, dovrebbe risalire al i 855 : si veda il «Bollettino bibliografico ebdomadario», I, 8 dicembre i 855 , 10, p. 79, numero 786. Un'edizione successiva, Il Bugiardello. L'oracolo degli antichi, Trieste, Editore Giovanni Peterlin, s.d. [ma I 900], recuperata dal mercato antiquario, è in mio possesso. Come soprannome, Bugiardello, è già attestato nella prima metà del Quattrocento in Toscana: si veda il Nanni di Piero di Ghinuccio chiamato Bugiardello, scovato da P. Ircani Menichini, Il quotidiano e i luoghi di Volterra nel catasto I429-I430, Volterra-Pisa, Migliorini, 2007, p. 24. 
8. G. Crocioni, Baldassarre da Fossombrone e il suo 'Menzoniero' o 'Bosadrello' alla corte dei Gonzaga, in «La Rinascita», XXXI (1943), pp. 224-57.

9. L'opera è conservata nella Biblioteca Nazionale Marciana di Venezia, con segnatura Misc. 4I 36.7. La disposizione dei sonetti appare basata su accostamenti tra testi che presentano affinità tematiche o lessicali, secondo un criterio presente nelle sillogi burchiellesche (al proposito M. Zaccarello, Introduzione a I sonetti del Burchiello, edizione critica della vulgata quattrocentesca a cura di M. Zaccarello, Bologna, Commissione per i testi di lingua, 2000, pp. XIII-CXXXII, a p. CXIX).

io. Fondamentale lo studio di C. MüLler-Fraureuth, Die deutschen Lügendichtungen bis auf Münchbausen, Hildesheim, Georg Olms Verlagsbuchhandlung, 1965 (reprint dell'ed. I88I). Tra le ultime pubblicazioni sul motivo si veda P. UHL, Un poème de menteries moyen-anglais: "Herkyn to My Tale", in «Alizés», $25-26$ (2005), pp. 245-57; per il versante inglese medievale utile N. Malcolm, The Origins of English Nonsense, London, Fontana Press, I997, pp. 58-62. Del genere si conoscono testimonianze in spagnolo: cfr. A. MARTínez PÉrez, La poesía medieval francesa del «non-sens»: Fatrasie y géneros análogos, Murcia, Universidad de Murcia, I 987, p. 68 n. 48 (sui limiti di questo lavoro la recensione di D. Musso, in «Cahiers de civilisation médiévale», XXXIII, I 29 (1990), pp. 77-80).

I I. La questione è ripercorsa da V. Molle, Les textes italiens du non-sens ( $X V^{e}$ XVI siècles) étudiés dans leurs rapports avec la littérature et les traditions orales, in Poésie et Rhétorique du non-sens. Littérature médiévale, littérature orale, sous la direction de S. Mougin et M.-G. Grossel, Reims, Presses Universitaires de Reims, 2004, pp. 239-73. Si veda anche $\mathrm{P}$. $\mathrm{BEC}$, De la «resverie» médiévale à la chanson traditionnelle des «menteries», in Atti del XIV congresso internazionale di linguistica e filologia roman₹a, a cura di A. Varvaro, vol. V, Napoli-Amsterdam, Macchiaroli-Benjamins, I98I, pp. $48 \mathrm{I}-89$.

I 2. G. Giannini, Canzoni alla rovescia, in «Rassegna nazionale», s. II, XXXVIII (I9I6), pp. 36-54.

I 3. F. Novati, Per la storia della Paremiografia Italiana ne' secoli XV e XVI. II. I proverbi del Farina, in «Il Libro e la Stampa», n.s., IV (1910), pp. 61-65.

I4. Tuttavia in una lettera di Ludovico Gonzaga, vescovo di Mantova (I44II 5 I I), a Ruffino Gablonetta, da Bracciano, in data I 3 giugno I 484, si trova una dichiarazione che farebbe pensare a un utilizzo ludico del testo già nella seconda metà del Quattrocento: «Le turche nostre tutte vogliamo presso di nui qui. Mandaticele adonque quanto più presto sia possibile, e cum esse li duoi libri de geomantia, lo grande e lo picolo, lo Bosadrello da li dati e li duoi libri da li Insomnii» (il passo della lettera è tratto dall'Archivio di Stato di Parma, Archivio Gonzaga di Guastalla, b. 41/3, reg. I, cc. I $78 \mathrm{v}-\mathrm{I} 79 \mathrm{r}$, pubblicato in D.S. CHAMBers, A Renaissance Cardinal and his worldly Goods: the Will and Inventory of Francesco Gonzaga (1444-1483), London, The Warburg Institute, I992, p. I 22 n. I42).

I 5. Cfr. Spagna ferrarese, I, 26-27: «Poi fece el re Marsilio raunare / tuta soa baronia in un giardino / e d'ariento se fece portare / apresso una fontana un gran baçino, / e una nave de cera fe' fare; / e davante a quel popul saracino / fece impire el 
baçino, et poi vi misse / la navicella, e poi legendo disse // per gramançia sopra el baçin più versi. / La nave subitamente se mosse / girando intorno in più modi diversi / ben decesete volte, e poi percosse / ne paesi di Spagnia et riversi. / Ive fermosse, poi perdé suo posse: / fiacossi tuta dov'era il porto scrito / in sul baçino sopra l'orlo drito» (ed. a cura di V. Gritti e C. Montagnani, Novara, Interlinea, 2009, pp. 235-36) e Iuli Valeri Res gestae Alexandri Macedoni, I, I: «quorum omnium Nectanabus prudentissimus fuisse comprobatur, quippe qui, quod alii armis, ille ore potuisse conuincitur. tantum denique sacricola peritia calluisse fertur ut mundialia quoque ei parerent elementa, adeo ut, si metus bellicus illi immineret, non exercitum, non machinamenta Martia moueret, quin potius ingressus aulae penita regiaeque secreta ibi se solitarium abdebat inuecta secum pelui. quam dum ex fonte liquidissimo impleret, ex cera imitabatur nauigii similitudinem effigiesque hominum illic collocabat. quae omnia cum supernare coepissent, mox moueri ac uiuere uisebantur. adhibebat etiam et uirgulam ex ligno ebeni et praecantamina loquebatur quibus uocaret deos superos inferosque sicque laborabat pelui nauiculam submergi. ex quo fiebat ut simul cum submersione illius cerae et cereis insessoribus etiam omnes hostes, si qui adesse praenuntiabantur, pelago mergerentur. itaque multo tempore regno ac secutirate potitus est» (in Il Romanzo di Alessandro, vol. I, a cura di R. Stoneman, trad. di T. Gargiulo, Milano, Fondazione Lorenzo Valla-Mondadori, 2007, p. 356 ).

I6. Rimando al mio contributo Un caso di poesia nonsensica secentesca: $i$ sonetti della "bugia" di Francesco Moise Chersino, in "Nominativi fritti e mappamondi». Il nonsense nella letteratura italiana. Atti del Convegno (Cassino, 9-10 ottobre 2007), a cura di G. Antonelli e C. Chiummo, Roma, Salerno Editrice, 2009, pp. I47-89.

17. Si veda G. Brunetti, Per il Romanzo d'Alessandro in Italia. Due poesie in un manoscritto dell'Historia de preliis (Leipzig, Universitätsbibliothek, Rep. II.4 ${ }^{\circ} .143$ ), in «Quaderni di Filologia romanza dell’Università di Bologna», XV (2001), pp. 379-90.

I 8. Per i volgarizzamenti della Lettera si vedano i numerosi studi di Lidia Bartolucci.

I9. Si veda G. Orlandi, Temi e correnti nelle leggende di viaggio dell'Occidente altomedievale, in Popoli e paesi nella cultura altomedievale, 2 to., Spoleto, Centro italiano di studi sull'alto medioevo, I983, to. II, pp. 523-71, M.S. MAzzi, Oltre l'orizzonte. In viaggio nel Medioevo, Cavallermaggiore, Gribaudo, I997, pp. 175-89 (cap. VI: Esseri disumani e meraviglie), e F. CARDINI, I viaggi immaginari, in Viaggiare nel Medioevo, a cura di S. Gensini, Pisa, Pacini, 2000, pp. 493-5 I6. Utile anche il volume di G. Bossi, Immaginario di viaggio e immaginario utopico. Dal sogno del paradiso in terra al mito del buon selvaggio, Milano, Mimesis, 2003.

20. Si veda A.R. Bartoli, La 'Navigatio Sancti Brendani' e la sua fortuna nella cultura romanza dell'età di mezzo, Fasano, Schena, I993, in part. pp. 353-94 (La Navigatio in Italia).

2 I. Cfr. T. Kohanski, The Book of John Mandeville. An edition of the Pynson Text with Commentary on the Defective Version, Tempe, Arizona Center for Medieval and Renaissance Studies, $200 \mathrm{I}$, p. vii. Il testo figura tra quelli conosciuti dal mugnaio Menocchio (cfr. C. Ginzburg, Il formaggio e $i$ vermi. Il cosmo di un mugnaio del 'soo, Torino, Einaudi, I999 ${ }^{2}$, p. 35 ). 
22. Con il titolo Il libro degli animali, si trova in circolazione una costosa riproduzione del codice, con annesso volume di commento a cura di C.M. Pyle (Milano, Jaca Book, 1985). Cfr. S. Cohen, Animals as Disguised Symbols in Renaissance Art, Leiden, Brill, 2008, pp. 23-25.

23. Si veda in particolare lo studio di E.M. Ackermann, Das Schlaraffenland in German Literature and Folksong. Social Aspects of an Earthly Paradise, with an inquiry into its History in European Literature, Chicago, University of Chicago, 1944, p. 67, che si serve di alcuni versi del Bugiardello stampato dal Pagan (e pubblicati da Rossi) per illustrare il motivo cuccagnesco.

24. Si leggano le considerazioni di C. KAPPLER, Demoni mostri e meraviglie alla fine del medioevo, trad. it., Firenze, Sansoni, I983, p. 52 . Il motivo è naturalmente diffuso anche nel XV sec.: valga l'esempio figurativo studiato da V. D. PINI, «Mundus inversus». Il mondo alla rovescia quale tema iconografico in una dimora quattrocentesca, in «I nostri monumenti storici», XXXVIII (1987), pp. 25 5-65.

25. Si veda F. Cardini, Prefazione a Kappler, Demoni, cit., pp. 7-I 2, a p. 9.

26. Sulla fortuna del Burchiello si rimanda al contributo di A. Decaria, Con Burchiello dopo Burchiello. Il 'nonsense' nella poesia toscana del secondo '400, in Nominativi fritti e mappamondi, cit., pp. 65-92.

27. Angelo Forte, Opera nuova. Dialogo primo, a cura di F. Lotti, in ID., Dialoghi letterari (Dialogo degli Incantamenti, Opera nuova), a cura di F. Troncarelli e F. Lotti, Manziana, Vecchiarelli, 201о, p. ı07; si veda anche F. Lотт, Erasmo, Luciano e l'alchimia: l'Opera nuova di Angelo Forte, in «Bruniana \& Campanelliana», XV (2009), 2, pp. 503-I 2.

28. Al proposito P. Mehtonen, Obscure Language, Unclean Literature: Theory and Practice from Quintilian to the Enlightenment, translated by R.MacGilleon, Helsinki, Finnish Academy of Science and Letters, 2003, pp. I 26-29.

29. Si veda Martínez Pérez, La poesía medieval francesa del «non-sens», cit., pp. I 84 e 206. Secondo G. Angel, Il senso del non-senso, in «Paragone», 27 (1976), 3 I 2, pp. 35-6 I, a p. 50, per quel che concerne l'esperienza fatrasica, questo tipo di attacco richiamerebbe le pastorelle.

30. G.R. Cardona, I viaggi e le scoperte, in Letteratura italiana, vol. V: Le Questioni, Torino, Einaudi, I986, pp. 687-716, a p. 705.

3 I. Cfr. I viaggi di Gio. da Mandavilla. Volgarizzamento antico toscano ora ridotto a buona lezione coll'aiuto di due testi a penna, per cura di F. Zambrini, 2 voll., Bologna, Romagnoli, i 870, vol. II, p. 63: «Ma non crediate, che io parli per ciancia, né per menzogna, avisandovi che io vidi cogli occhi miei canne sì grandi sopra queste rive, che xx. de' nostri compagni non poterono levare una sola da terra»; si legga anche ivi, p. 95: «E quantunque alcuni sieno che non credono, e tengono a favola e bugia quel ch'io discrivo della nobilità di sua persona, di suo stato, di suo cortesia e del grande ordine di gente che tiene, niente dimeno io dirò alcuna parte delle maniere e dell'ordine di lui e della sua gente, sicondo che io ho veduto molte volte. Chi mi vuol credere, mi creda, se gli piace». 
32. In Visioni dell'aldilà in Occidente. Fonti Modelli Testi, a cura di M.P. Ciccarese, Firenze, Nardini, I987, p. 52; si veda Kappler, Demoni, cit., p. 32.

33. M. BARSI, Postfazione. «Non è creduta, e pur piace». Riflessioni sull'iperbole in retorica (XVI-XX secolo), in Funzioni e finzioni dell'iperbole tra scienze e lettere, a cura di M. Barsi e G. Boccali, Milano, Cisalpino, 20 Iо, pp. 3 I 5-49, a p. 3 I6.

34. Si veda I. Kozminsky, I numeri magici. Simbolismo, significato e usi quotidiani, trad. it., Roma, Tattilo, I973, pp. 34-39, Kappler, Demoni, cit., p. 89, e R.A. Laroche, Popular Symbolic/Mystical Numbers in Antiquity, in «Latomus», 54 (1 995), pp. 568-76, a p. 57 I. Un esempio in Inf. XXII, I03.

35. Cfr. A. Natale, Gli specchi della paura. Il sensazionale e il prodigioso nella letteratura di consumo (secoli XVII-XVIII), Roma, Carocci, 2008, pp. I99-203; da ultimo M. ANGELINI, Le meraviglie della generazione. Voglie materne, nascite straordinarie e imposture nella storia della cultura e del pensiero medico in età moderna (secoli XV-XIX), Milano, Mimesis, $20 \mathrm{I} 2$.

36. Si veda U. Pizzani, I 'monstra' nella cultura classica, in I 'monstra' nell'inferno dantesco: tradizione e simbologie. Atti del XXXIII Convegno storico internazionale (Todi, I 3-I 6 ottobre 1996), Spoleto, Centro Italiano di Studi sull'Alto Medioevo, I997, pp. I-26, a p. I 2 e n. 57. Giuseppe Cremascoli ricorda che in un poema di Ras-Shamra del XIV sec. a. C. si legge «tu schiaccerai Leviatàn, serpente fuggiasco, tu consumerai il serpente tortuoso, il potente dalle sette teste» (G. Cremascoli, L'immaginario dei 'monstra' biblici, in I 'monstra' nell'inferno dantesco: tradizione e simbologie, cit., pp. 27-4I, a p. 37).

37. Cfr. J.-L. HaquetT, Iperbole e alterità. Riflessioni sul gigante nella finzione letteraria, in Funzioni e finzioni dell' iperbole tra scienze e lettere, cit., pp. 106- I 9. In particolare, in XXIV, I 6- I 7: «qui son giganti alti mille braza / e ben un miglio è longa la lor faza», le dimensioni del viso di questi giganti sembrano voler marcare un'evidente distanza da Dante, Inf. XXXI, 58-59: «La faccia sua mi parea lunga e grossa / come la pina di San Pietro a Roma».

38. Cfr. G. Chiesa Isnardi, La figura del fabbro sourumano nella letteratura nordica antica, in Il superuomo e i suoi simboli nelle letterature moderne, prefazione di E. Zolla, vol. V, Firenze, La Nuova Italia, I977, pp. I I-40.

39. Si veda la coda: «Questa non fu busia, / in fe' de lial omo el ver ti narro: / el portò via l'oche, i bovi e 'l carro». L'immagine è quasi certamente ricalcata su un passo di John Mandeville, il quale racconta di grifoni che possono rapire in volo due buoi legati ad un carro: cfr. JEAn de MandeVIlle, Le livre des merveilles du monde, édition critique par Ch. Deluz, Paris, CNRS, 2000, XXIX, p. 431: «Mes un griffoun ad le corps plus grant et plus fort qe $\mathrm{VIII}^{\circ}$ leouns, de leouns par decea, et plus de grandesse et de force qe $\mathrm{C}$ aigles, qar il emporte bien a soun ny en volant un grant chival et le mene sus s'il troeve au point, ou II boefs lyez ensemble si qe homme [les lie] a la charue» (si veda anche Kappler, Demoni, cit., p. IIG).

40. Per iperboli affini nelle fatrasies si veda ANGeli, Il senso del non-senso, cit., pp. 4I-42. Di fatto le due scene di Baldassarre richiamano quella del grifone che 
solleva l'elefante, nell'episodio menzionato da Marco Polo (cfr. Kappler, Demoni..., cit., p. 57).

4I. Cito dall'ed. a cura di V. Branca, 2 voll., Torino, Einaudi, I992, vol. II, p. I 222 e n. 3 per gli antecedenti dei viaggi miracolosi; cfr. inoltre M. Montesano, «Fantasima, fantasima che di notte vai». La cultura magica nelle novelle toscane del Trecento, prefazione di G. Airaldi, Roma, Città Nuova, 2000, p. 46. Da ultima É. ZANNONE, Le vol magique de messire Torello dans l'édition illustrée du Décaméron (I492, Venise, Gregorio e Giovanni De Gregori), in «Cahiers d'études italiennes. Filigrana», I 2 (2010), pp. 37-52.

42. Si veda Il viaggio di rabbi Petachiah di Ratisbona, a cura di I. Ventrice, Firenze, Giuntina, 2009, I6, p. 41: «Gli fecero vedere un cammello volante, basso e dalle gambe gragili. Se qualcuno vuole galopparci sopra deve legarsi ad esso per non cadere. Ciò che un uomo percorre in quindici giorni, il cavaliere sul cammello volante lo percorre in un giorno. Nello stesso tempo potrebbe percorrere ancora più strada, ma nessuno resisterebbe ad un galoppo così veloce, perché in un istante salta già un miglio» (cfr. A. TOAFF, Mostri giudei. L'immaginario ebraico dal Medioevo alla prima età moderna, Bologna, Il Mulino, I996, p. 23). Come osserva la curatrice in nota, si tratta dello Strutbio camelus (su cui M. Scarabelli, Il "cammello" e il falso pellegrino. Chiose su Ècci venuto Guido ['n] Compostello di Niccola Muscia, in «Lettere italiane», LXI (2009), I, pp. I I0-26, in part. pp. I I 5-1 7); il passo del racconto ebraico è stato citato anche da Mazzi, Oltre l'orizzonte, cit., p. 178. La bibliografia sul motivo del viaggio favoloso si recupera dall'annotazione di Branca (Boccaccio, Decameron, cit., vol. II, pp. I 205-6).

43. In Poeti del Duecento, a cura di G. Contini, 2 to., Milano-Napoli, Ricciardi, I960, to. I, p. 642 .

44. Per il Burchiello cito dall'ed. a cura di M. Zaccarello, Torino, Einaudi, 2004. Soluzioni simili in Fatrasies d'Arras, I, 8: «Et cler jor par nuit oscure» e Resveries, I 4: «Par un matin me levai / Quant il fu nuis» (in Poésies du non-sens. XIII ${ }^{e}-X I V^{e}$ $X V^{*}$ siècles. Tome II: Resveries, textes édites, traduits et commentés par M. Rus, Orléans, Paradigme, 2010, p. 43).

45. Cfr. W. Fauth, Carmen magicum. Das Thema der Magie in der Dichtung der römischen Kaiserzeit, Frankfurt am Main, Lang, I999, pp. I I7-I 8.

46. Cito dall'ed. contenuta nel volume Poésies du non-sens. XIII -XIV $X V^{\mathcal{E}}$ siècles. Tome I: Fatrasies (Fatrasies de Beaumanoir - Fatrasies d'Arras), textes édités, traduits et commentés par M. Rus, Orléans, Paradigme, 2005 (=FB e $F A$ ), p. 54; nessuna nota di commento sul verso. Cfr. anche Martínez Pérez, La poesía medieval francesa del «non-sens», cit., p. 27: «No aparecen monstruos extraños ni exóticos, lo más raro a lo que se llega es al "ours emplumés" (F.A. LIV), a la "truie enceinte" (F.A. XLVI), al "Dragons de geline" (F.A. XIX)» (e ivi, p. I 5 ). Daniela Musso vedeva nella creazione del sintagma Dragons de geline un accostamento dovuto a «un'identità fonica in uno o più punti» (D. Musso, La «logica» del nonsenso. A proposito di fatrasie $e$ fatras, in «Lingua e letteratura», IX, I7 (I99I), pp. 7I-82, a p. 77). Giovanna Angeli parla di «une dualité animale, aussi irréductible qu'inexplicable», specificando più avanti che «Le "dragon de geline” repré- 
sente le résultat d'un croisement anormal: la bête fabuleuse que nous a transmise un imaginaire archaïque pourrait avoir été pondue par une poule. Mais il est plus vraisemblable que le vers de l'auteur arrageois veuille juste nous peindre une créature fantastique mêlant les attributs du dragon à ceux d'un gallinacé» (G. Angeli, Un monde à part: la fatrasie et les images dans les marges, in L'allusion dans la littérature. Actes du XXIV ${ }^{\mathrm{e}}$ Congrès de la Società Universitaria per gli Studi di Lingua e Letteratura Francese (SUSLLF), En Sorbonne, novembre 1998, textes réunis par M. Murat, Paris, Presses de l'Université de Paris-Sorbonne, 2000, pp. $25-40$, a p. 28.

47. Cito dall'ed. a cura di M.L. Colker, Patavii, in aedibus Antenoreis, I978, p. 268.

48. Andrea Cappellano, Trattato d'amore. Andreae Capellani Regii Francorum "De amore" libri tres, testo latino del sec. XII con due traduzioni toscane inedite del sec. XIV, a cura di S. Battaglia, Roma, Perrella, i 947, p. I 44. Cfr. B.N. SARGENTBAur, Alexander and the "Conte du Graal", in Arthurian Literature, XIV, ed. by J. P. Carley and F. Riddy, Cambridge, Brewer, I996, pp. I-18, a p. I6 n. 30. In una glossa all'Alexandreis nel ms. Laur. Strozz. I 38 si legge «In ortu Alexandri, ut asserit (lege: asserunt) hystoriographi, multa miracula apparuerunt: peperit autem in eadem die gallina draconem» (R. DE CESARE, Glosse latine e antico francesi all" " $A$ lexandreis" di Gautier de Châtillon, Milano, Vita e Pensiero, I95 I, p. I3 n. I). L'immagine è inclusa anche in Iuli Valeri Res gestae Alexandri Macedoni, I, I I: «Enimuero $\dagger$ pauens $\dagger$ cum in quadam regiae Philippus sessitaret in qua aues plurimae circumerrarent isque intentus agendis rebus animum occupauisset, repente gallina supersiliens eius in sinum considensque enixa est ouum. sed ouum illud euolutum sinu eius humi concrepat, cuiusque testula dissultante dracunculus utpote $\langle\mathrm{e}\rangle$ tantillo conclaui pertenuis egredi uisitur isque saepe circumcursans et ambiens oui testulam uelle se rursus eo unde emerserat condere» (in Il Romanzo di Alessandro, vol. I, cit., p. 372).

49. Cfr. Giovanni Battista Ardemani, Il tesoro delle gioie [...], Venezia, Ciotti, i6o2, LIII, p. $22 \mathrm{I}$.

50. Cfr. A. VAsina, voce "Classe", in Enciclopedia dantesca, Roma, Istituto della Enciclopedia Italiana, I984², 6 voll., vol. II, pp. 29-30. Sulla pineta di Ravenna nel medioevo si veda P. FABBri - A. Missiroli, Le pinete ravennati. Storia di un bosco e di una città, Ravenna, Longo, I998, pp. 9-23.

5 I. Per i frutti antropomorfi si rimanda a Kappler, Demoni, cit.,pp. I 2 I-22.

52. Al proposito P.V.Ch. Baur, Centaurs in ancient Art. The archaic Period, Berlin, Curtius, ı 91 2, pp. I I0-30; sul cervo la bibliografia si può recuperare da E. Ferrarini, Sicut cervus. Esegesi patristica ed altomedievale di PS 4I (42), 2, in Studi in onore di Gilberto Lonardi, a cura di G. Sandrini, Verona, Fiorini, 2008, pp. I 57-82. Sui centauri armati si veda Biblioteca Medicea Laurenziana. La Biblioteca in mostra. Animali fantastici, Firenze, Mandragora, 2007, pp. 28-29; si veda anche ivi, pp. 52-53, e 64-65. 
53. Sui sagittari nella cultura medievale F. DuBost, Aspects fantastiques de la littérature narrative médiévale (XII'-XIII siècles). L'Autre, l'Ailleurs, l'Autrefois, Paris, Champion, I99I, pp. 527-39.

54. Forte, Opera nuova. Dialogo terzo, cit., p. I 39. John Mandeville raccontava di una pianta che al posto dei consueti frutti produceva uccelli (cfr. KaPpler, Demoni, cit., pp. 59-60). In LVI, I 2-I7 Baldassarre scrive del celebre «agnello vegetale» (si veda ancora KAPPLER, Demoni, cit., pp. I I9-20; per i frutti zoomorfi, ivi, pp. I 20-2I).

55. L'iconografia della sirena musicante non è estranea alla cultura classica: si veda S. Ensoli, Le Sirene omeriche e le Sirene musicanti di età classica, in Ulisse: Il mito e la memoria (Roma, Palazzo delle Esposizioni, 22 febbraio-2 settembre I996), Roma, Progetti museali, i996, pp. 96-107.

56. Cito dall'ed. a cura di M. Bonafin, Alessandria, Edizioni dell'Orso, 2007 , pp. 76 e 78. Sul tema G. PERON, Meraviglioso e verosimile nel romanzo francese medievale: da Benoît de Sainte-Maure a Jean Renart, in Il meraviglioso e il verosimile tra antichità e medioevo, a cura di D. Lanza e O. Longo, Firenze, Olschki, I989, pp. 293-323, in part. pp. 296-303 e P. DiBie, Storia della camera da letto. Il riposo e l'amore nei secoli, trad. it., Milano, Bompiani, 2005 , p. 87.

57. Croce parla di «garbusi genovesi», 'cavoli genovesi' (in Giulio Cesare Croce, L'Eccellenza e Trionfo del Porco e altre opere in prosa, a cura di M. Rouch. Giornata tranquilla, a cura di F. Bacchelli, con una prefazione di F. Foresti, Bologna, Pendragon 2006, pp. 233-47, a p. 239).

58. Viazo da Venesia al Sancto Iherusalem, a cura di A. e F. Petrucci, Roma, Edizioni dell'Elefante, i972, p. 80. Giordano da Séverac racconta che nell'attuale Ceylon: «Sunt arbores quaedam quae habent folia ita magna quod possint stare quinque vel sex homines, valde bene, sub umbra illius» (in KAPPLER, Demoni, cit., p. IO I n. 44).

59. Va precisato che l'arcangelo Gabriele figura come guida fino al Paradiso nel Libro della Scala: cfr. E. Ceruldi, Il "Libro della Scala" e la questione delle fonti arabospagnole della 'Divina Commedia', Città del Vaticano, Biblioteca Apostolica Vaticana, i949, pp. 63-22 I. Si veda anche Chanson de Roland, vv. 2395-396: «Ensemble'od els sent Gabriel i vint: / L'anme del cunte portent en pareïs» (ed. a cura di M. Bensi, introduzione di C. Segre, trad. di R. Lo Cascio, testo antico francese a fronte, Rizzoli, Milano i98 5, p. 340).

6o. Cfr. W.R. Lethaby, Architettura, misticismo e mito, trad. it., Bologna, Pendragon, 2003, pp. 85-Io8 (cap. V: L'albero dei gioielli). Si tratta, naturalmente, anche di un motivo fiabesco: M. Rak, Logica della fiaba [...], Milano, Bruno Mondadori, 2005, p. 153 .

6. Angelo Clareno, Cronica della tribulazioni, in Mistici del Duecento e del Trecento, a cura di A. Levasti, Milano, Rizzoli, i960, pp. 319-36, a p. 33 I.

62. Dall'ed. a cura di A.E. Quaglio, Milano, Mondadori, I998, p. I I 3. 
63. Forte, Opera nuova. Dialogo teræo, cit., p. I40.

64. Cfr. Lethaby, Architettura, misticismo e mito, cit., p. 94. Si veda anche il caso di Odorichus, De rebus incognitis. Odorico da Pordenone nella prima edizione a stampa del i5 I3, a cura di L. Monaco - G.C. Testa, Pordenone, Camera di Commercio di Pordenone, I986, 26, I4-I 5, p. I I 7: «Et anchora ve dirò magiore maraviglie, che in quella contrada anchor nasce una generatione de canne non più grosse che se sia le nostre de qua [...], e vanno per nove vie a questo modo più de un grosso miglio; et è piena de nodi, como le canne nostre: ogne nodo per sotto terra fa la radice. E dicivano fermamente che infra queste canne se ne trova assaissime che hanno doi e qual tre pietre preciose de grossezza de una fava, et ha de singularissime virtude».

65. Per la forma Camalech si veda Jean de Mandeville, Le livre des merveilles $d u$ monde, cit., XXV, p. 397.

66. Forte, Opera nuova. Dialogo ter₹o, cit., pp. I4I-42. Come precisa la curatrice a pp. 91-92, il Dialogo terzo era stato incluso da Imbriani nella Novellaja fiorentina (cfr. l'ed. con cronologia e nota introduttiva di I. Sordi, Milano, Rizzoli, I997, pp. 359-74); cfr. anche V. Rossi nell'Appendice II ad Andrea Calmo, Lettere, a cura di V. Rossi, Torino, Loescher, I 888, pp. 401-2 e n. I (di p. 402).

67. In Bestiari medievali, a cura di L. Morini, Torino, Einaudi, I 996, p. 248. Si veda anche Francesco Bracciolini, Scherno de gli dèi: XIX, xx, 6, p. 406: «Diverran vili, come una Cicogna» (LO SCHERNO | DE GLI DEI | Poema | DEL SIG. BRACCIOLINO | Dell'Api | Con l'aggiunta di sei Canti, | et altre rime piacevoli | Dell'istesso AVtore. [...] | In Roma, Per il Mascardi. mDcxxvi. |Ad instanza di Giouanni Manelfi | Con licenza de' Superiori., p. 402; esemplare consultato: Roma, Biblioteca Nazionale Centrale, con segnatura 623 F 50 ).

68. Il verbo si provaro è tecnico, di ambito militare, e significa 'combatterono': si veda al proposito Spagna ferrarese, II, 35, I-2: «Datime la parola, madre mia, / ch'io vadi al campo a provarmi cum loro» (dall'ed. cit., p. 257 e n. ad locum).

69. Cfr. W. Deonna, La grenouille et le lion, in «Bullettin de Correspondence Hellénique», LXXVIV (1950), pp. I-9. Al v. I6 dello stesso sonetto IV or ora citato, "se non che l'antiguardia sen fu accorto», è ipotizzabile che sia stato omesso un titulus sull'ultima $a$ di antiguardia; si potrebbe avere così, più coerentemente, antiguardian, che meglio si accorderebbe con accorto: si veda Spagna ferrarese, XIX, I6, 6: «che per antiguardian ognun abatte» (ed. cit., p. 552 ).

70. Su questo motivo paradossale si veda Angeli, Un monde à part, cit., pp. 34-38.

7 I. L'aspetto temibile dell'oca è noto alla simbologia celtica: si veda S. HeINz, I simboli dei Celti. Il fascino magico di un popolo straordinario, Vicenza, Il punto d'incontro, $2008^{3}$, p. I I9: «Oltre alle caratteristiche degli altri animali d'acqua, l'oca può essere molto aggressiva, cosa che la mette in relazione con i combattenti». Non ho potuto prendere visione del lavoro di F. Unterkircher, Tiere, Glaube, Aberglaube. Die schönsten Miniaturen aus dem Bestiarium, Graz, Akademische Druck-u. Verlagsanstalt, I 986. 
72. Anche in questo caso ci troviamo in presenza di una variante del motivo del rovesciamento dei ruoli tra la lepre e i cani (Angeli, Un monde à part, cit., p. 30).

73. Alla mansione sono preposti i cani, come si deduce anche dal sonetto XX, I- 5 .

74. Il sintagma "re di corona" significa 'vero re' (cfr. Spagna ferrarese, VIII, 45, 3: «Magiarigi e 'l figlinol, re di corona», dall'ed. cit., p. 373 e n.). Si noti il richiamo incongruo all'auctoritas, espediente tipicamente burchiellesco. L'incoronamento degli animali parrebbe motivo comico: si veda Angeli, Un mond à part, cit., pp. 32-33.

75. Cfr. FA 53, I: «Blanche robe noire».

76. Heinz, I simboli dei Celti, cit., pp. I 25-27 e i 29.

77. Odorichus, De rebus incognitis, 22, 24 (ed. cit., p. ior). Un corvo parlante si trova anche nei Sonetti del Burchiello, cit., LXXIII, 9- I , p. Iо3: «Odi che fantasia venne a un corbo / che contendeva collo dio d'amore / dicendogli "Superbo, ingrato et orbo"». Sui corvi parlanti si veda anche F. MASPERO, Bestiario antico [...], Casale Monferrato, Piemme, i997, pp. I 22-23.

78. Cecco D'Ascoli, L'Acerba, XXIV, I-2, in Bestiari medievali, cit., p. 596 e n. a p. 623 con richiamo a Bartolomeo Anglico.

79. Cfr. Teofilo Folengo, Zanitonella V, 926-28: «Hor la, comenza. Sed, o vadit tot mundus atornum! / Quo casamenta volant? Quo caprae? Quo mea vacca? / Quo mea vacca, inquam, volat alta viamque menatur?» (in ID., Macaronee minori. Zanitonella - Moscheide - Epigrammi, a cura di M. Zaggia, Torino, Einaudi, I987, p. 275).

80. Cfr. D. MORris, Le civette e altre creature della notte. Storia, mito e realtà di un mistero nato insieme all'uomo, trad. it., Roma, Castelvecchi, 2009, p. 48; si veda anche Kappler, Demoni..., cit., p. 36. Per il luogo non proprio pacifico si rimanda a G. Cremascoli, L'immaginario dei 'monstra' biblici, cit., p. 29 e nn. Forse i versi di Baldassarre sono stati all'origine di quelli di Giulio Cesare Croce, Sogni fantastichi della notte [...], Bologna, Benacci, I600, c. A3v: «Molte volte ho sonato la Trombetta, / Il Trombon, e la Piva, e la soffiare / Son doventato un Gufo, o una Civetta».

8I. Fra i tanti saggi sull'argomento citiamo D. PAQuetTe, Les figurations à thème musical sur les chapiteux romans, in «Annales de l'Académie de Macon», VI (1994), pp. I4I-48 e C. PANTI, Animali musicanti fra ilarità, etica e studio dell'anima: il lupo violista e la questione «Utrum sciencia sit de numero bonorum» del ms. Siena, Biblioteca Comunale L. III. 2I, in Homo risibilis: capacità di ridere e pratica del riso nelle civiltà medievali. Atti del convegno (Siena, 2-4 ottobre 2002), a cura di F. Mosetti Casaretto, Alessandria, Edizioni dell'Orso, 2005, pp. 367-88.

82. Sul primo verso si veda $F B$ I, 7: «Uns muiau i vint chanter», $F A$ 5, 7: «Uns muiaus dit qu'il ont tort» e $F A 48$, 9: «Qui fist parler deus muiaus». Su questo tipo di opposizioni è intervenuta ANGeLI, Il senso del non-senso, cit., pp. 40-4I. 


\section{Giuseppe Crimi}

83. Si legga Babio, 45 5-56: «Quando miser sapies? Nec erit citharedus asellus, / Nec bos docta loquens, nec Babio sapiens» (dall'ed. a cura di A. Dessì Fulgheri, in Commedie latine del XII e XIII secolo, II, Genova, Università di Genova-Istituto di filologia classica e medievale, I980, p. 296). L'immagine era percepita come un adynaton, ma era leggibile anche come evento prodigioso: un mago, nel Floire et Blanceflor, insegna a un asino a suonare l'arpa (segnalazione di E. DE LA WARR Bejamin, A Study of the Magic in the Romans d'Aventure and the Romans Bretons, part I, Baltimore, Furst, I 906, p. I 5 , ripresa da R. Kieckhefer, La magia nel Medioevo, trad. it., Roma-Bari, Laterza, I993, p. г 26).

84. Si veda E. SCARPa, Un'ipotesi sulla morte di Margutte, in «Filologia e critica», I (1976), pp. I 38-44.

8 5. Bartoli, La 'Navigatio Sancti Brendani' e la sua fortuna nella cultura romanza dell'età di mezzo, cit., pp. I 35-37. Sugli animali giganti Kappler, Demoni, cit., p. I I6.

86. Si legge in Ceruldi, Il "Libro della Scala", cit., p. 99.

87. Odorichus, De rebus incognitis, io, 6 (ed. cit., p. 64).

88. Cfr. Poggio Bracciolini, De varietate fortunae, IV, 226-29: «Frequentissimus apud hos ludus est galli inuicem pugnantes. Hos diuersi producunt ad pugnam, quisque suum superaturum asserens, proque alterius uictoria pecuniam etiam adstantes inuicem ponunt» (edizione critica con introduzione e commento a cura di O. Merisalo, Helsinki, Suomalainen Tiedeakatemia, I993, p. I62).

89. Si veda A. Arioli, Le città mirabili. Labirinto arabo medievale, Milano, Mimesis, 2003.

90. Bartoli, La 'Navigatio Sancti Brendani' e la sua fortuna nella cultura romanz̧a dell'età di mezzo, cit., p. 379.

9i. Al proposito A. Cattaneo, Scritture di viaggio e scrittura cartografica. La mappamundi di fra Mauro e i racconti di Marco Polo e Niccolò de' Conti, in «Itineraria», 3-4 (2004-2005), pp. I 57-202, alle pp. I 87-89.

92. Oddorichus, De rebus incognitis, i 5,7 (ed. cit., pp. 73 e 76).

93. Cfr. Dubost, Aspects fantastiques de la littérature narrative médiévale, cit., pp. 35 I-89 (e n. Io a pp. 888-89, nella quale si tratta dello splendido palazzo di Costantinopoli) e BARTOLI, La 'Navigatio Sancti Brendani' e la sua fortuna nella cultura romanża dell'età di mezzo, cit., p. 87: "Il cristallo è il materiale per eccellenza dell'Altro Mondo: quando le bansídhe si manifestano ad un mortale sotto l'aspetto di fanciulle e gli chiedono di seguirlo, di solito sono a bordo di un curach di cristallo. Queste caratteristiche d'impalpabilità e fragilità che inizialmente servivano a rendere l'eccezionalità del materiale e la natura magica del palazzo si trasformano, nella letteratura cortese più matura, in preziosità e ricchezza (metalli nobili e gemme)». Nelle Mille e una notte si racconta di un castello di cristallo (in KaPpLER, Demoni, cit., p. 77).

94. Jean de Mandeville, Le livre des merveilles du monde, cit., XXV, p. 389. 
95. Oddorichus, De rebus incognitis, i6, i 8-20 (ed. cit., p. 80); si veda anche ivi, 23, 7-8: «A questo idolo ogni anno si gli fa una solennissima festa, et al tempo de la festa illi mettono questo idolo de oro in uno carro de legno alho $<\mathrm{e}>$ bellissimo quanto se po': et ha octo rote et è ornato con tanti drappi d'oro e prete preciose e perle che credo che nel mondo non ne sia altratante e de sì gran valuta» (ivi, p. 105$)$.

96. Si legga almeno JeAn de MandevilLe, Le livre des merveilles du monde, cit., XXVI, p. 405 .

97. Cfr. N. Miller, Medieval Garden Fountains, in Medieval Gardens. Dumbarton Oaks Colloquium on the History of Landscape Architecture, edited by E.B. MacDougall, Washington, Dumbarton Oaks Trustees for Harvard University, I986, pp. I 35-53, in part. I 38-4I.

98. Si veda A. Delatte, La catoptromancie grecque et ses dérivés, Liège-Paris, VaillantCarmanne-Droz, 1932 (in part. pp. 48-6i per il XV sec.) e S. Melchior-Bonnet, Storia dello specchio, trad. it., Bari, Dedalo, 2002, pp. 238-42. Mi preme rilevare, nel sonetto, il passaggio dal fons imventutis allo specchio magico, commistione che sembra di origine orientale: «Nella tradizione islamica è appunto un re persiano, Gamshid, il quale possiede una coppa nel fondo della quale si può vedere l'universo intero» (F. CARDINI, L'acqua come simbolo nel giardino toscano tardomedievale, in «Mélanges de l’École française de Rome. Moyen Âge», I 04 (1992), 2, pp. 545-53, a p. 548).

99. Recupero i versi da R. Palgen, La 'Visione di Tundalo' nella 'Commedia' di Dante, in «Convivium», a. XXXVII (1969), 2, pp. I 29-47, a pp. I37-38.

ıоo. Cito dall'ed. a cura di P. Scopelliti e A. Chaouech, Milano, Mimesis, 2006, pp. 77-78.

io I. Per l'ultimo verso soccorre B. VAn den Abeele, Le faucon sur la main. Un parcours iconographique médiéval, in La Chasse au Moyen Age. Société, traités, symboles. Textes réunis par A. Paravicini Bagliani et B. Van den Abeele, Firenze, SismelEdizioni del Galluzzo, 2000, pp. 87-109 (in part. pp. 107-9 per la simbologia negativa).

io2. Cfr. Jacopo da Verona, Liber Peregrinationis, VII: «Prope Kayrum ad X miliaria. est locus. ubi nascitur balsamum. et est ibi unus fons et unus puteus. de cujus aqua rigatur ille ortus. qui est longus modicum plusquam ad jactum baliste et latus modicum minus: et arbor faciens balsamum parva est» (ed. a cura di U. Monneret de Villard, Roma, La Libreria dello Stato, I950, p. 84).

I03. Cfr. G. Busi, Qabbalah ebraica, Torino, Einaudi, 2005, p. 25 r: «L'idea di accostare olio buono e balsamo deriva da un versetto del profeta Isaia, in cui è detto che il re Ezechia mostrò agli inviati del re di Babilonia il balsamo e l'olio buono ...che si trovava nei suoi magazzini (Is 39.2). Lo spunto biblico si unisce poi a un'immagine di origine midrašica, secondo la quale nell'aldilà scorrono fiumi di balsamo. "Quando i giusti stanno per morire - si legge infatti nel midraš Genesi rabbah - Dio mostra loro la ricompensa che li attende. Nell'attimo in cui rabbi 
Abbahu era sul punto di spirare, gli furono mostrati tredici fiumi di balsamo"» (corsivo nel testo).

i04. Si veda Aurelius Prudentius Clemens, Liber Cathemerinon, V, i I 7-i 8: «Illic [scil. in patria iustorum] et gracili balsama surculo / desudata fluunt», in Visioni dell'aldilà in Occidente, cit., p. 62.

ios. Lapo Gianni, Amor, eo chero mia donna in domino, vv. I-2, in Poeti del Duecento, cit., to. II, p. 603 .

Io6. Cfr. JACOPO DA Verona, Liber peregrinationis, VII,: «et recipitur magnum theloneum de omnibus infinitis camelis transeuntibus: et audivi a Saracenis et Christianis. quod quasi infinitam pecuniam valet illud theloneum» (ed. cit., p. 87).

i07. Si veda Caio Giulio Solino, Da Roma a Taprobane, Dai Collectanea rerum memorabilium, a cura di B. Basile, Roma, Carocci, 2010, XI, 43-44, p. I 38 : «Sola India mittit avem psittacum colore viridem torque puniceo, cuius rostri tanta duritia est, ut cum e sublimi praecipitat in saxum, nisu se oris excipiat et quodam quasi fundamento utatur extraordinariae firmitatis»; sono gli uccelli che figurano anche nella corte del Gran Can (Jean De Mandeville, Le livre des merveilles $d u$ monde, cit., XXV, p. 395).

io8. Jean de Mandeville, Le livre des merveilles du monde, cit., XXV, p. 398.

io9. Caio Giulio Solino, Da Roma a Taprobane, cit., XI, 50-5 I, p. I 40.

I Io. Descrizione in C. ANgeleri, Bibliografia delle stampe popolari a carattere profano dei secoli XVI e XVII conservate nella Biblioteca Nazionale di Firenze, Firenze, Sansoni Antiquariato 1953, numero 64, pp. 71-72. L'opuscolo non era sfuggito agli studiosi della Scuola storica: si veda Pietro Bembo, Motti, a cura di V. Cian, premessa di A. Gnocchi, nota al testo e indici a cura di G. Raboni, Milano, Sylvestre Bonnard, 2007, p. 98.

I I I. Si leggono in M. CHIESA, Un anonimo raffinato per un letteratissimo poemetto popolareggiante, in Il Parnaso e la zucca. Testi e studi folenghiani, a cura di M. Chiesa e S. Gatti, Alessandria, Edizioni dell'Orso, I995, pp. 103-18, alle pp. i I4-I 5.

I I 2. Cfr. L. Emery, $I l$ Lamento e la Farza de li massare di Velardiniello, in «Archivio storico per le province napoletane», n.s., XXII (1936), pp. 324-40, in part. p. 335 dove sono riprodotti i versi 4-6 e 8 dal sonetto III (dal testo offerto da Rossi).

I I 3. Riproduco i versi da Velardiniello e la sua inedita farsa napoletana. Memoria letta all'Accademia Pontaniana nella tornata del s dicembre 1909 dal socio Benedetto Croce, in «Atti dell'Accademia Pontaniana», XL (I910), 2, pp. I-24, pp. i9-20.

I I 4. Ho consultato l'esemplare della Biblioteca Universitaria Alessandrina, con segnatura Let. Pop. A.8; sia reso grazie alla dott.ssa Saveria Rito per la disponibilità e per la cortesia. Dell'opuscolo si conoscono alcune stampe antecedenti (rispetto a quella da me consultata), ossia Firenze, presso Francesco Spiombi i 842; Prato, A spese di M. Contrucci, I 86 I (cfr. G. Giannini, Canti popolari toscani, 
presentazione di M. Luzi, introduzione di C. Lapucci, Palermo, Edikronos, I98 I, pp. 290-9I; un esemplare è conservato presso la Biblioteca Riccardiana di Firenze, con segnatura N.A.U i66, un secondo a Weimar, Hersogin Anna Amalia Bibliothek, con segnatura Koe 1 54 I [c]; e un terzo a Strasbourg, Bibliothèque Nationale Universitaire, con segnatura CD. ı 04.748 ,2, 6), e Firenze, Salani, I 870. Un'edizione Firenze, Salani, i 882, viene segnalata da Vittorio Rossi in Calmo, Le Lettere, cit., p. 449, n. 2.

I I 5. Strambottoli, cit., 3, p. 4. Sul motivo si rimanda a Giannini, Canzoni alla rovescia, cit.

I I6. Strambottoli, cit., 7, p. 5 .

I I 7. Il campanile di cristallo di Baldassarre sembra memore della torre di cristallo descritta nella Navigatio Sancti Brendani: si veda Bartol, La 'Navigatio Sancti Brendani' e la sua fortuna nella cultura romanza dell'età di mezzo, cit., pp. 86-88 e i90-98. Un'altra immagine degna di attenzione è quella di Strambottoli, 5,7 : «Era fratello di un Gigante nano» e $F A$ 36, 7: «Uns grans homs / qui estoit nains».

i i 8. Cfr. Carlo Goldoni, Il poeta fanatico, a cura di M. Amato, Venezia, Marsilio, I996, III, I, pp. I92-93; III, 2, p. I94; III, 7, p. 200 (la segnalazione di uno dei passi goldoniani si legge in P. MicheLi, Letteratura che non senso, Livorno, Giusti, I900, p. 52); in particolare, l'attacco dello strambottolo I6, Era di notte e non ci si vedea, richiama un verso del Furioso (XL, 6, I), come precisa G. Fumagaldi, Chi l'ha detto? [...], Milano, Hoepli, I $995^{10}$, p. I 54 , numero 568. Per questo burchiellismo settecentesco si rinvia a M. CAtucci, Burchielli e tartane, in «Sincronie», VI (2002), I I, Pp. I $50-55$.

i 9. Gianni Rodari, Il libro degli errori (1964), in Id., I cinque libri. Storie fantastiche, favole, filastrocche, disegni di B. Munari, Torino, Einaudi, $2009^{15}$, p. 45 I. Si leggano le riflessioni di G. Massini, La poetica di Rodari. Utopia del folklore e nonsense, Roma, Carocci, 20I I, pp. 75-I 50 (Rodari, poeta nonsense?). 